\title{
Joint Analysis of Health Histories, Physiological State, and Survival
}

\author{
Anatoly I. Yashin \\ Igor Akushevich \\ Konstantin G. Arbeev \\ Alexander Kulminski \\ Svetlana Ukraintseva \\ Center for Population Health and Aging, Duke University, \\ Durham, North Carolina
}

Data on individual health histories, age trajectories of physiological or biological variables, and mortality allow for the study of the joint evolution of health and physiological states and their effects on mortality. Individual health and physiological trajectories are described using a stochastic process with two mutuallydependent continuous and jumping components. The parameters of this process and mortality rate are identified from the data in which the continuous component is measured in discrete times, and transitions of jumping process are observed.

Keywords: aging related changes; longitudinal data; mortality modelling; observational plans

\section{INTRODUCTION}

In order to characterize the mortality rate in terms of parameters describing aging-related decline in health and physiological and biological functioning, one has to specify the conditional mortality rate as a function of health status and physiological or biological state and describe the age-specific changes in both of these components. Changes in individual health or in survival are usually described using finite state continuous time random Markov process with

Address correspondence to Igor Akushevich, Center for Population Health and Aging (CPHA), Duke University, Trent Drive Hall \#001 (Box 90408), Durham, NC 27708, USA. E-mail: igor.akushevich@duke.edu 
age-dependent transition intensities. The absorbing state is death, and transition intensities to this state are mortality rates from disease states. Some of the determinants of health transitions are fixed at the time of birth, and others vary over the life course (physiological state, behavioral, or socio-economic factors.) In the presence of stochastic time-varying factors affecting transition rates, the jumping process ceases to have the Markov property. This complicates the description of aging related changes in health and survival.

The connection between continuously changing aging-related components and mortality were studied by Strehler and Mildvan (1960), Sacher and Trucco (1962) (review by Yashin et al., 2000), but all of these authors had only mortality data. Woodbury and Manton (1977) suggested a stochastic process model to analyze longitudinal data, relying on the conditional Gaussian property of the distribution of physiological variables among survivors. The model describes physiological changes using stochastic differential equations, and the respective stochastic processes are Markov (Yashin, 1980, 1985; Yashin and Manton, 1997; Akushevich et al., 2005; Yashin et al., 2007, 2008; Arbeev et al., 2009). Bagdonavicius and Nikulin (2009) introduced "degradation modelling" into the analysis of failure time data using different ideas. None of these models described changes in health status accompanying physiological aging.

When health is taken into account, the coefficients of the stochastic differential equations describing physiological aging depend on the jumping process describing changes in health. Covariates are no longer Gaussian and do not have the Markov property. We suggest a comprehensive model of human aging, health, and mortality where jumping and continuous components jointly have the Markov property. The jumping component stands for fast changes in health status, and the continuous component describes slower individual physiological aging.

We show that the parameters of this model can be identified from incomplete longitudinal data, representing results of discrete measurements of physiological variables and changes in individuals' health status.

\section{MODEL}

The finite-state jumping stochastic process $\theta_{t}(t \geq 0), \theta_{t} \in\{1,2, \ldots, M\}$, where $M$ is the total number of states, describes changes in health status. The $K$-dimensional stochastic process $Y_{t}(t \geq 0)$ with continuous components represents the changes of physiological variables with age. $Y_{t}$ satisfies a stochastic differential equation with coefficients 
depending on $\theta_{t}$ :

$$
d Y_{t}=A_{\theta_{t}}\left(Y_{t}, t\right) d t+b_{\theta_{t}}(t) d W_{t}
$$

with initial conditions $Y_{t_{0}}=Y_{0}, A_{\theta_{t}}\left(Y_{t}, t\right)$ is a vector of dimension $K$, $b_{\theta_{t}}(t)$ is a $K \times K$-matrix, and $W_{t}$ is a $K$-dimensional Wiener process with independent components and independent of $Y_{t_{0}}$.

The finite state continuous time process $\theta_{t}$ is characterized by the conditional transition intensity matrix from state $k$ to state $r$ :

$$
\lambda_{k r}\left(Y_{t}, t\right), \quad k, r=1,2, \ldots, M, \quad \text { and } \quad \lambda_{k k}\left(Y_{t}, t\right)=-\sum_{r=1, r \neq k}^{M} \lambda_{k r}\left(Y_{t}, t\right)
$$

and initial probabilities $P\left(\theta_{t_{0}}=j\right), j=1,2, \ldots, M$.

Death at time $T$ means that the trajectories of $\theta_{t}$ and $Y_{t}$ are stopped at time $T$. The conditional distribution of $T$ given the trajectories of $\theta_{u}$, $Y_{u}, 0 \leq u \leq t$ is given by the conditional hazard rate $\mu_{\theta_{t}}\left(Y_{t}, t\right)$. The triple $\left(\theta_{t}, Y_{t}, T\right)$ describes the aging of an individual. $N$ individuals whose health status and physiological state are described by $\theta_{t}$ and $Y_{t}$ have life spans $T_{1}, \ldots, T_{N}$. The likelihood function is:

$$
L\left(T_{1}, T_{2}, \ldots, T_{N}\right)=\prod_{i=1}^{N} \overline{\bar{\mu}}\left(T_{i}\right)^{\delta_{i}} \exp \left(-\int_{0}^{T_{i}} \overline{\bar{\mu}}(u) d u\right)
$$

with

$$
\overline{\bar{\mu}}(t)=E\left(\mu_{\theta_{t}}\left(Y_{t}, t\right) \mid T>t\right),
$$

the total mortality rate and $\delta_{i}$ the censoring variable. In traditional survival analysis the likelihood function in Eq. (3) has to be maximized with respect to parameters of $\overline{\bar{\mu}}(t)$. The hazard rate $\overline{\bar{\mu}}(t)$ can seldom be expressed in terms of parameters relevant to $\theta_{t}$ and $Y_{t}$. The maximization then requires solving nonlinear partial differential and ordinary differential equations.

To calculate $\overline{\bar{\mu}}(t)$ in Eq. (4), one needs the functional form of the conditional mortality rate $\mu_{\theta_{t}}\left(Y_{t}, t\right)$ and

$$
f(y, j \mid t)=\frac{\partial}{\partial y} P\left(Y_{t} \leq y, \theta_{t}=j \mid T>t\right),
$$

which is the joint conditional probability density function (p.d.f.) with respect to $Y_{t}$, and the probability with respect to $\theta_{t}$, given $T>t$. According to Bayes rule $f(y, j \mid t)=\psi(y, j, t) / P(T>t)$, where function $\psi(y, j, t)=\frac{\partial}{\partial y} P\left(Y_{t} \leq y, \theta_{t}=j, T>t\right)$ satisfies the 
Kolmogorov-Fokker-Plank equation:

$$
\begin{aligned}
\frac{\partial}{\partial t} \psi(y, j, t)= & \sum_{i=1}^{M} \lambda_{i j}(y, t) \psi(y, j, t)-\frac{\partial}{\partial y}\left(A_{j}(y, t) \psi(y, j, t)\right) \\
& +\frac{1}{2} \frac{\partial^{2}}{\partial y^{2}}\left(B_{j}(t) \psi(y, j, t)\right)-\psi(y, j, t) \mu_{j}(y, t) .
\end{aligned}
$$

Taking into account that $P(T>t)$ satisfies:

$$
\frac{d}{d t} P(T>t)=-\overline{\bar{\mu}}(t) P(T>t),
$$

we get the partial differential equation for $f(y, j \mid t)$ (Yashin et al., 1995):

$$
\begin{aligned}
\frac{\partial}{\partial t} f(y, j \mid t)= & \sum_{i=1}^{M} \lambda_{i j}(y, t) f(y, i \mid t)-\frac{\partial}{\partial y}\left(A_{j}(y, t) f(y, j \mid t)\right) \\
& +\frac{1}{2} \frac{\partial^{2}}{\partial y^{2}}\left(B_{j}(t) f(y, j \mid t)\right)+f(y, j \mid t)\left(\overline{\bar{\mu}}(t)-\mu_{j}(y, t)\right)
\end{aligned}
$$

with initial conditions $f\left(y, j \mid t_{0}\right) . A_{j}(y, t)$ are defined in Eq. (1) and $B_{j}(t)=b_{j}(t) b_{j}(t)^{*}$ (* denotes transposition). Transition intensities $\lambda_{k r}(y, t), k, r=1,2, \ldots, M$ are defined by Eq. (2). Because $f(y, j \mid t)$ multiplies $\overline{\bar{\mu}}(t)$ in Eq. (8), and $\overline{\bar{\mu}}(t)$ is the result of integration of $\mu_{\theta_{t}}\left(Y_{t}, t\right)$ with respect to $f(y, j \mid t)$, Eq. (8) is a nonlinear partial integral-differential equation with respect to $f(y, j \mid t)$.

The total mortality rate $\overline{\bar{\mu}}(t)$ is also:

$$
\overline{\bar{\mu}}(t)=\sum_{j=1}^{M} \bar{\mu}_{j}(t) \pi_{j}(t)
$$

where $\pi_{j}(t)=P\left(\theta_{t}=j \mid T>t\right)$, and

$$
\bar{\mu}_{j}(t)=E\left(\mu_{\theta_{t}}\left(Y_{t}, t\right) \mid \theta_{t}=j, T>t\right) .
$$

To calculate Eq. (9) and (10), one needs $\pi_{j}(t)=P\left(\theta_{t}=j \mid T>t\right)$ and the conditional p.d.f. $f(y \mid j, t)=\partial P\left(Y_{t} \leq y \mid \theta_{t}=j, T>t\right) / \partial y$ for each $t \geq 0$. $\pi_{j}(t)$ is derived by integrating $f(y, j \mid t)$ in Eq. (8) with respect to $y$ :

$$
\frac{d \pi_{j}(t)}{d t}=\sum_{k=1}^{M} \bar{\lambda}_{k j}(t) \pi_{k}(t)+\pi_{j}(t)\left(\overline{\bar{\mu}}(t)-\bar{\mu}_{j}(t)\right), \quad j=1,2, \ldots M
$$


with initial conditions $\pi_{j}\left(t_{0}\right)$. Here $\overline{\bar{\mu}}(t)$ and $\bar{\mu}_{j}(t)$ are given by Eq. (9) and (10), and $\bar{\lambda}_{i j}(t)$ is defined as:

$$
\bar{\lambda}_{i j}(t)=E\left(\lambda_{i j}\left(Y_{t}, t\right) \mid \theta_{t}=i, T>t\right)=\int_{R^{K}} \lambda_{i j}(y, t) f(y \mid i, t) d y .
$$

Integration in Eq. (10) and (12) requires $f(y \mid j, t), j=1,2, \ldots, M$. The equation for this conditional p.d.f. results from Bayes' rule and from Eq. (8) and (11):

$$
\begin{aligned}
\frac{\partial}{\partial t} f(y \mid j, t)= & \sum_{i=1}^{M}\left(\lambda_{i j}(y, t) f(y \mid i, t)-\bar{\lambda}_{i j}(t) f(y \mid j, t)\right) \frac{\pi_{i}(t)}{\pi_{j}(t)} \\
& -\frac{\partial}{\partial y}\left(A_{j}(y, t) f(y \mid j, t)\right)+\frac{1}{2} \frac{\partial^{2}}{\partial y^{2}}\left(B_{j}(t) f(y \mid j, t)\right) \\
& +f(y \mid j, t)\left(\bar{\mu}_{j}(t)-\mu_{j}(y, t)\right)
\end{aligned}
$$

with initial conditions $f\left(y \mid j, t_{0}\right)$. Eq. (11) and (13) is solved numerically.

To solve Eq. (11) and (13), the functional forms for the conditional transition intensities $\lambda_{k r}\left(Y_{t}, t\right)$, for the conditional mortality rate $\mu_{\theta_{t}}\left(Y_{t}, t\right)$ in Eq. (10) and (12), and for coefficients $A_{\theta_{t}}\left(Y_{t}, t\right)$ and $B_{\theta_{t}}(t)$ in Eq. (8) and (13), must be specified. We take quadratic forms of $Y_{t}$ :

$$
\begin{aligned}
& \lambda_{k r}\left(Y_{t}, t\right)=\lambda_{0 k r}(t)+\left(Y_{t}-g_{k}(t)\right)^{*} \Lambda_{k r}(t)\left(Y_{t}-g_{k}(t)\right), \\
& \mu_{\theta_{t}}\left(Y_{t}, t\right)=\mu_{0 \theta_{t}}(t)+\left(Y_{t}-f_{\theta_{t}}(t)\right)^{*} Q_{\theta_{t}}(t)\left(Y_{t}-f_{\theta_{t}}(t)\right) .
\end{aligned}
$$

$\Lambda_{k r}(t)$ and $Q_{\theta_{t}}(t)$ are symmetric nonnegative definite $K \times K$ matrices, $f_{\theta_{t}}(t)$ and $g_{k}(t)$ are $K$-vector functions, and $\lambda_{0 k r}(t)$ and $\mu_{0 r}(t)$ are parametric functions of $t$, for $k, r, j=1,2, \ldots, M$, for $t \geq t_{0}$. In application to analysis of physiological trajectories it is convenient to modify Eq. (1) using $A_{\theta_{t}}\left(Y_{t}, t\right)=a_{\theta_{t}}(t)\left(Y_{t}-f_{1 \theta_{t}}(t)\right)$ :

$$
d Y_{t}=a_{\theta_{t}}(t)\left(Y_{t}-f_{1 \theta_{t}}(t)\right) d t+b_{\theta_{t}}(t) d W_{t}
$$

with initial conditions $Y_{t_{0}} . a_{\theta_{t}}(t)$ is a matrix function of negative feedback coefficients, and components of the vector function $f_{1 \theta_{t}}(t)$ characterize the effects of allostatic adaptation on physiological state (Yashin et al., 2007, 2008). All other notations are the same as in Eq. (1). According to Eq. (16), the trajectories of $Y_{t}$ will tend to follow $f_{1 \theta_{t}}(t)$. How close these curves are depends on the absolute values of feedback coefficients $a_{\theta_{t}}(t)$ and the magnitude of disturbances determined by coefficients $b_{\theta_{t}}(t)$.

When coefficients in Eq. (15) and (16) do not depend on a jumping process $\theta_{t}$ and when the distribution for initial value $Y_{t_{0}}$ is Gaussian, 
the conditional probability distribution of the process $Y_{t}$ among survivors to age $t$ is also Gaussian (Yashin, 1980, 1985; Yashin and Manton, 1997). The dependence of these coefficients on $\theta_{t}$ makes $Y_{t}$ no longer Gaussian. Therefore, we use the Gaussian approximation of the conditional p.d.f. $f(y \mid j, t)=\partial P\left(Y_{t} \leq y \mid \theta_{t}=j, T>t\right) / \partial y$.

The conditional hazard given health status $\theta_{t}=j$, and the unconditional transition intensity functions are:

$$
\begin{aligned}
& \bar{\mu}_{j}(t)=\mu_{0 j}(t)+\left(m_{j}(t)-f_{j}(t)\right)^{*} Q_{j}(t)\left(m_{j}(t)-f_{j}(t)\right)+\operatorname{Tr}\left(Q_{j}(t) \gamma_{j}(t)\right), \\
& \bar{\lambda}_{j k}(t)=\lambda_{0 j k}(t)+\left(m_{j}(t)-g_{j}(t)\right)^{*} \Lambda_{j k}(t)\left(m_{j}(t)-g_{j}(t)\right)+\operatorname{Tr}\left(\Lambda_{j k}(t) \gamma_{j}(t)\right),
\end{aligned}
$$

where

$$
\left.m_{j}(t)=E\left(Y_{t} \mid \theta_{t}=j, T>t\right)\right)
$$

and

$$
\left.\gamma_{j}(t)=E\left(\left(Y_{t}-m_{j}(t)\right)\left(Y_{t}-m_{j}(t)\right)^{*} \mid \theta_{t}=j, T>t\right)\right) .
$$

These conditional moments satisfy (see Appendix A):

$$
\begin{gathered}
\frac{d m_{j}(t)}{d t}=\sum_{i=1}^{M} \frac{\pi_{i}(t)}{\pi_{j}(t)}\left(m_{i j}(t) \bar{\lambda}_{i j}(t)-2 \gamma_{i}(t) \Lambda_{i j}(t) \check{\boldsymbol{g}}(t)\right) \\
-a_{j}(t) \check{f}_{1 j}(t)+2 \gamma_{j}(t) Q_{j}(t) \check{f}_{j}(t), \\
\frac{d \gamma_{j}(t)}{d t}=\sum_{i=1}^{M} \frac{\pi_{i}(t)}{\pi_{j}(t)}\left(\left(\gamma_{i}(t)-\gamma_{j}(t)+m_{i j}(t) m_{i j}^{*}(t)\right) \bar{\lambda}_{i j}(t)\right. \\
\left.+2\left(\gamma_{i}(t) \Lambda_{i j}(t) \gamma_{i}(t)-\gamma_{i}(t) \Lambda_{i j}(t) \check{g}_{i}(t) m_{i j}^{*}(t)-m_{i j}(t) \check{g}_{i}^{*}(t) \Lambda_{i j}(t) \gamma_{i}(t)\right)\right) \\
+a_{j}(t) \gamma_{j}(t)+\gamma_{j}(t) a_{j}^{*}(t)+B_{j}(t)-2 \gamma_{j}(t) Q_{j}(t) \gamma_{j}(t)
\end{gathered}
$$

$\bar{\lambda}_{i j}(t)$ is given by Eq. (18), $m_{i j}(t)=m_{i}(t)-m_{j}(t), \check{f}_{j}(t)=f_{j}(t)-m_{j}(t)$, $\check{f}_{1 j}(t)=f_{1 j}(t)-m_{j}(t), \check{g}_{i}(t)=g_{i}(t)-m_{i}(t)$.

\section{OBSERVATIONAL PLANS}

The parameters describing such a mortality model are many, and the survival data alone may not be sufficient to identify them all. This is why additional data on aging-related changes are needed. Such data are usually incomplete, and their structure reflects measurements scheme specified in the study design. For example, physiological 
trajectories could not be entirely observed during individuals' lives and are usually represented by a sequence of measurements performed at discrete time points. Individuals' health can also be measured at discrete time points. Alternatively, health transitions can be observed when they occur during the life course. Such different systems of measurements are called "observational plans." Distinguishing among such plans is important because it determines the functional form of the likelihood function of the data used in the parameter estimation procedure and affects the accuracy of parameter estimates. We distinguish three observational plans. Plan 1 contains discrete-time observations of continuously changing physiological variables. The changes in health status are unobserved. In Plan 2, physiological variables are not measured, but health transitions are observed. Plan 3 combines Plans 1 and 2. It is represented by a sequence of measurements of the physiological state and by a sequence of health transitions.

\subsection{Plan 1. Continuous Variables are Observed at Discrete Times, no Measurement of Health State}

Continuously changing variables are measured at ages $t_{0}<t_{1}$ $<t_{2} \ldots<t_{n} \leq T . \widetilde{Y}_{0}^{t}=Y_{t_{0}}, Y_{t_{1}}, Y_{t_{2}}, \ldots, Y_{t_{n}}$ is a random vector of observations at these ages. $\widetilde{Y}_{0}^{t_{k-}}=\widetilde{Y}_{0}^{t_{k-1}}$ and $\widetilde{Y}_{0}^{t}=\widetilde{Y}_{0}^{t_{k}}$, if $t_{k} \leq t<t_{k+1}$. Here $t_{k}-=\lim _{u \uparrow t_{k}} t_{u}$.

The conditional probability of having health status $j$, given $\widetilde{Y}_{0}^{t}$, and $T>t$ is:

$$
\tilde{\pi}_{j}(t)=P\left(\theta_{t}=j \mid \widetilde{Y}_{0}^{t}, T>t\right),
$$

and

$$
\tilde{f}(y \mid j, t)=\frac{\partial}{\partial y} P\left(Y_{t} \leq y \mid \widetilde{Y}_{0}^{t}, \theta_{t}=j, T>t\right)
$$

is the conditional probability density function of $Y_{t} . \tilde{\pi}_{j}(t)$ and $\tilde{f}(y \mid j, t)$ start at age $t_{0}$, and vary over the intervals $\left[t_{i}, t_{i+1}\right), i=0, \ldots, n-1$, satisfying Eq. (11) and (13), respectively. $\tilde{\pi}_{j}(t)$ and $\tilde{f}(y \mid j, t)$ both jump at the observation times $t_{1}, t_{2}, \ldots, t_{n}$. The values of these functions after the jumps follow from standard Bayes arguments:

$$
\tilde{\pi}_{j}\left(t_{k}\right)=\tilde{\pi}_{j}\left(t_{k}-\right) \frac{\tilde{f}\left(Y_{t_{k}} \mid j, t_{k}-\right)}{\sum_{r=1}^{M} \tilde{\pi}_{r}\left(t_{k}-\right) \tilde{f}\left(Y_{t_{k}} \mid r, t_{k}-\right)}, \quad \tilde{f}\left(y \mid j, t_{k}\right)=\delta\left(y-Y_{t_{k}}\right) .
$$


Here $\tilde{\pi}_{j}\left(t_{k}-\right)=\lim _{t \uparrow t_{k}} \tilde{\pi}_{j}(t)$ and

$$
\tilde{f}\left(Y_{t_{k}} \mid j, t_{k}-\right)=\frac{\partial}{\partial y} P\left(Y_{t} \leq y \mid \widetilde{Y}_{0}^{t-}, \theta_{t}=j, T>t\right)_{t=t_{k}, y=Y_{t_{k}}} .
$$

They are solutions of Eq. (11) and (13) over the interval $\left[t_{k-1}, t_{k}\right)$ with $\bar{\lambda}_{k j}(t)$ replaced by $\tilde{\bar{\lambda}}_{k j}\left(\widetilde{Y}_{0}^{t}, t\right), \overline{\bar{\mu}}(t)$ by $\tilde{\bar{\mu}}\left(\widetilde{Y}_{0}^{t}, t\right)$, and $\bar{\mu}_{j}(t)$ by $\tilde{\mu}_{j}\left(\widetilde{Y}_{0}^{t}, t\right)$. Transition coefficients are:

$$
\tilde{\bar{\lambda}}_{k j}\left(\widetilde{Y}_{0}^{t}, t\right)=E\left(\lambda_{k j}\left(Y_{t}, t\right) \mid \widetilde{Y}_{0}^{t}, \theta_{t}=k, T>t\right),
$$

the mortality rate is:

$$
\tilde{\bar{\mu}}\left(\widetilde{Y}_{0}^{t}, t\right)=\sum_{j=1}^{M} \tilde{\mu}_{j}\left(\widetilde{Y}_{0}^{t}, t\right) \tilde{\pi}_{j}(t),
$$

and

$$
\tilde{\mu}_{j}\left(\widetilde{Y}_{0}^{t}, t\right)=E\left(\mu_{\theta_{t}}\left(Y_{t}, t\right) \mid \widetilde{Y}_{0}^{t}, \theta_{t}=j, T>t\right) .
$$

The values of Eq. (25) serve as initial conditions for modified Eq. (11) and (13) over the interval $\left[t_{k}, t_{k+1}\right)$. The likelihoods include data on the sequences of discrete-time measurements of continuously changing component (e.g., physiological state) for each study participant, in addition to survival data. The component of the likelihood function dealing with discrete-time measurements involves the conditional p.d.f.: $\tilde{\phi}(y \mid t)=\partial P\left(Y_{t} \leq y \mid \widetilde{Y}_{0}^{t}, T>t\right) / \partial y$, which is represented as $\tilde{\phi}(y \mid t)=\sum_{r=1}^{M} \tilde{f}(y \mid r, t) \pi_{r}(t)$. The second component describes survival data and involves the conditional mortality rate $\tilde{\mu}_{j}\left(\widetilde{Y}_{0}^{t}, t\right)$. The component of the likelihood function for the $i$-th individual having measurements $y_{t_{1}^{i}}^{i}, y_{t_{2}^{i}}^{i}, \ldots, y_{t_{n(i)}^{i}}^{i}$ and $T_{i}$ is:

$$
\begin{aligned}
& L_{i}\left(y_{t_{1}^{i}}^{i}, y_{t_{2}^{i}}^{i}, \ldots, y_{t_{n(i)}^{i}}^{i}, T_{i}\right) \\
& =\tilde{\phi}\left(y_{t_{n(i)}^{i}}^{i} \mid t_{n(i)}^{i}-\right) \tilde{\phi}\left(y_{t_{n(i)-1}^{i}}^{i} \mid t_{n(i)-1}^{i}-\right) \ldots \tilde{\phi}\left(y_{t_{1}^{i}}^{i} \mid t_{1}^{i}-\right) \\
& \quad \times \tilde{\tilde{\mu}}^{i}\left(T_{i}\right)^{\delta_{i}} \exp \left(-\int_{0}^{T_{i}} \tilde{\bar{\mu}}^{i}(u) d u\right) .
\end{aligned}
$$

Here $\tilde{\phi}\left(y_{t_{k}^{i}}^{i} \mid t_{k}^{i}-\right)=\sum_{r=1}^{M} \tilde{f}\left(y_{t_{k}^{i}}^{i} \mid r, t_{k}^{i}-\right) \pi_{r}\left(t_{k}^{i}-\right)$, and $\delta_{i}$ indicates censoring for the $i$-th individual. To maximize the likelihood function of Eq. (30), one should solve modified Eq. (11) and (13) for different values of intermediate parameter estimates at each step of the likelihood 
maximization procedure. Solving a system of nonlinear partial differential equations (PDE) at each iteration step is computationally prohibitive. To reduce the computational load, one could use the Gaussian approximation of the conditional probability density function $\tilde{f}(y \mid j, t)$, and solve systems of ordinary differential equations for the first two moments of this distribution, instead of solving the PDE given by Eq. (13).

\subsubsection{Gaussian Approximation of $\tilde{\boldsymbol{f}}(\boldsymbol{y} \mid \boldsymbol{j}, t)$ in the Case of Plan 1}

In the case of the quadratic mortality risk, quadratic transition intensity functions, and linear equations for $Y_{t}$, the likelihood function of Eq. (30) is approximated as:

$$
\begin{aligned}
L_{i}\left(y_{t_{0}^{i}}^{i}, y_{t_{1}^{i}}^{i}, \ldots, y_{t_{n_{i}}^{i}}^{i}, T_{i}\right)= & \tilde{\bar{\mu}}^{i}\left(\tilde{y}_{0}^{T_{i}-}, T_{i}\right)^{\delta_{i}} \exp \left(-\int_{0}^{T_{i}} \tilde{\bar{\mu}}^{i}\left(\tilde{y}_{0}^{i u}, u\right) d u\right) \\
& \times \prod_{j=0}^{n_{i}}\left(\sum_{k=1}^{M} \tilde{\pi}_{k}^{i}\left(t_{j}^{i}-\right) \chi_{k}\left(y_{t_{j}^{i}}^{i}, t_{j}^{i}-\right)\right),
\end{aligned}
$$

where

$$
\chi_{k}(y, t)=\left(2 \pi\left|\tilde{\gamma}_{k}^{i}(t)\right|\right)^{-\frac{K}{2}} \exp \left(-\frac{1}{2}\left(y-\tilde{m}_{k}^{i}(t)\right)^{*} \tilde{\gamma}_{k}^{i}(t)^{-1}\left(y-\tilde{m}_{k}^{i}(t)\right)\right),
$$

$\tilde{\bar{\mu}}\left(\tilde{y}_{0}^{i t}, t\right)$ is defined in Eq. (28), $\tilde{y}_{0}^{T_{i-}} \equiv \tilde{y}_{0}^{t^{i}(i)}$, and $\tilde{\mu}_{j}\left(\tilde{y}_{0}^{i t}, t\right)$ is represented as:

$$
\tilde{\mu}_{j}\left(\tilde{y}_{0}^{i t}, t\right)=\mu_{0 j}(t)+\left(\tilde{m}_{j}^{i}(t)-f_{j}(t)\right)^{*} Q_{j}(t)\left(\tilde{m}_{j}^{i}(t)-f_{j}(t)\right)+\operatorname{Tr}\left(Q_{j}(t) \tilde{\gamma}_{j}^{i}(t)\right)
$$

with

$$
\left.\tilde{m}_{k}^{i}(t)=E\left(Y_{t} \mid \tilde{y}_{0}^{i t}, \theta_{t}=k, T_{i}>t\right)\right)
$$

and

$$
\left.\tilde{\gamma}_{k}^{i}(t)=E\left(\left(Y_{t}-\tilde{m}_{k}^{i}(t)\right)\left(Y_{t}-\tilde{m}_{k}^{i}(t)\right)^{*} \mid \tilde{y}_{0}^{i t}, \theta_{t}=k, T_{i}>t\right)\right) .
$$

The transition intensities $\tilde{\lambda}_{k j}\left(\widetilde{Y}_{0}^{t}, t\right)$ in Eq. (11) for $\tilde{\pi}_{j}(t)$ are $\tilde{\lambda}_{k j}\left(\widetilde{Y}_{0}^{t}, t\right)=\lambda_{0 k j}(t)+\left(\tilde{m}_{k}(t)-g_{k}(t)\right)^{*} \Lambda_{k j}(t)\left(\tilde{m}_{k}(t)-g_{k}(t)\right)+\operatorname{Tr}\left(\Lambda_{k j}(t) \tilde{\gamma}_{k}(t)\right)$.

In Eq. (31), $\delta_{i}$ is a censoring indicator, $K$ is the dimension of vector $Y_{t}, \tilde{m}_{k}^{i}(t)$ and $\tilde{\gamma}_{k}^{i}(t)$ satisfy Eq. (21) and (22) over the intervals $\left[t_{0}^{i}, t_{1}^{i}\right) ;\left[t_{1}^{i}, t_{2}^{i}\right) ; \ldots ;\left[t_{n(i)-1}^{i}, t_{n(i)}^{i}\right) ;\left[t_{n(i)}^{i}, T_{i}\right)$ with the initial conditions 
$y_{t_{0}^{i}}^{i}, y_{t_{1}^{i}}^{i}, \ldots, y_{t_{n(i)}^{i}}^{i}$ for $\tilde{m}_{k}^{i}\left(t_{j}^{i}\right)=y_{t_{j}^{i}}^{i}$, and $\tilde{\gamma}_{k}^{i}\left(t_{j}^{i}\right)=0$, respectively; $\tilde{m}_{k}^{i}\left(t_{j}^{i}-\right)$ $=\lim _{t \uparrow t_{j}^{i}} \tilde{m}_{k}^{i}(t)$, and $\tilde{\gamma}_{k}^{i}\left(t_{j}^{i}-\right)=\lim _{t \uparrow t_{j}^{i}} \tilde{\gamma}_{k}^{i}(t)$, and $t_{n(i)}^{i}$ is the age of the latest measurement of the physiological index before death at $T_{i}$ for the $i$-th individual. Using index $i$ in $\tilde{\pi}_{k}^{i}(t), \tilde{m}_{k}^{i}(t)$, and $\tilde{\gamma}_{k}^{i}(t)$ in Eq. (11), (21), and (22) is necessary because the values of these estimates depend on individual sequence of measurements of the process $Y_{t}$.

The conditions of Eq. (25) are expressed as:

$$
\tilde{\pi}_{j}\left(t_{i}\right)=\tilde{\pi}_{j}\left(t_{i}-\right) \frac{\chi_{j}\left(Y_{t_{i}}, t_{i}-\right)}{\sum_{k=1}^{M} \tilde{\pi}_{k}\left(t_{i}-\right) \chi_{k}\left(Y_{t_{i}}, t_{i}-\right)}
$$

with $\tilde{m}_{j}\left(t_{i}\right)=Y_{t_{i}}$ and $\tilde{\gamma}_{j}\left(t_{i}\right)=0 . \chi_{j}(y, t)$ is defined in Eq. (32).

The dynamics of $\tilde{\pi}_{j}(t)$ follows from Eq. (11) with $\tilde{\lambda}_{k j}\left(\tilde{Y}_{0}^{t}, t\right)$ used instead of $\bar{\lambda}_{k j}(t)$ over the intervals $\left[t_{l}, t_{l+1}\right), l=0, \ldots, n-1$. The initial values of $\tilde{\pi}_{j}(t)$ at the beginning of $l$-th interval $\left[t_{l}, t_{l+1}\right)$ are given by the relationship which involves the values of $\tilde{\pi}_{j}\left(t_{l}-\right), \tilde{m}_{j}\left(t_{l}-\right)$, and $\tilde{\gamma}_{j}\left(t_{l}-\right)$ which are solutions of Eq. (11), (21), and (22) at the end of the interval $\left[t_{l-1}, t_{l}\right)$.

\subsection{Plan 2. Measuring Changes in Health State. No Measurements of Physiological State}

The equation for conditional probability density function

$$
\widehat{f}(y, t)=\frac{\partial}{\partial y} P\left(Y_{t} \leq y \mid \theta_{0}^{t}, T>t\right)
$$

of $Y_{t}$, given age trajectories of health history $\theta_{0}^{t}$, and $T>t$ is

$$
\begin{aligned}
\frac{\partial}{\partial t} \widehat{f}(y, t)= & -\frac{\partial}{\partial y}\left(A_{\theta_{t}}(y, t) \widehat{f}(y, t)\right)+\frac{1}{2} \frac{\partial^{2}}{\partial y^{2}}\left(B_{\theta_{t}}(t) \widehat{f}(y, t)\right) \\
& +\widehat{f}(y, t)\left(\sum_{k=1, k \neq \theta_{t-}}^{M} \widehat{\lambda}_{\theta_{t-}, k}(t)-\sum_{k=1, k \neq \theta_{t-}}^{M} \lambda_{\theta_{t-}, k}(y, t)\right) \\
& +\widehat{f}(y, t)\left(\widehat{\mu}_{\theta_{t-}}(t)-\mu_{\theta_{t-}}(y, t)\right) .
\end{aligned}
$$

Eq. (39) has to be solved over the intervals $\left[t_{0}, \tau_{1}\right),\left[\tau_{1}, \tau_{2}\right),\left[\tau_{2}, \tau_{3}\right)$, $\ldots,\left[\tau_{m}, T\right)$, between subsequent jumps of the process $\theta_{t}$, where $\tau_{1}$, $\tau_{2}, \ldots, \tau_{m}$ are ages at which changes in a person's health status took place (dates of jumps of the process $\theta_{t}$ ). To avoid multiple hierarchical indexing we denote $\theta_{t} \equiv \theta(t)$. The initial conditions at the beginning of each interval $\left[\tau_{1}, \tau_{2}\right),\left[\tau_{2}, \tau_{3}\right), \ldots,\left[\tau_{m}, T\right)$ are 


$$
\widehat{f}\left(y, \tau_{p}\right)=\widehat{f}\left(y, \tau_{p}-\right) \frac{\lambda_{\theta\left(\tau_{p}-\right), \theta\left(\tau_{p}\right)}\left(y, \tau_{p}-\right)}{\widehat{\lambda}_{\theta\left(\tau_{p}-\right), \theta\left(\tau_{p}\right)}\left(\tau_{p}-\right)} .
$$

Here $\widehat{f}\left(y, \tau_{p}-\right)=\frac{\partial}{\partial y} P\left(Y_{\tau_{p}} \leq y \mid \theta_{0}^{\tau_{p-1}}, T>\tau_{p}\right)$ is solution of Eq. (39) over the interval $\left[\tau_{p-1}, \tau_{p}\right)$ at the time just before the $p$-th jump of the process $\theta_{t}$ at time $\tau_{p}$. Here and below the sign comma in $\lambda_{\theta\left(\tau_{p}-\right), \theta\left(\tau_{p}\right)}$ stands to separate two aggregative indices of $\lambda$.

$$
\begin{gathered}
\widehat{\lambda}_{\theta\left(\tau_{p}-\right), \theta\left(\tau_{p}\right)}\left(\tau_{p}\right)=\left.E\left(\lambda_{\theta\left(\tau_{p}-\right), x}\left(Y_{\tau_{p}}, \tau_{p}\right) \mid \theta_{0}^{\tau_{p}-}, T>\tau_{p}\right)\right|_{x=\theta\left(\tau_{p}\right)}, \\
\widehat{\mu}_{\theta(t)}(t)=E\left(\mu_{\theta(t)}\left(Y_{t}, t\right) \mid \theta_{0}^{t}, T>t\right) .
\end{gathered}
$$

The likelihood for Plan 2 corresponding to ages of change in the health status (age at onset of diseases) for the $i$-th individual with $m(i)$ changes in the health status happened at time points $\tau_{1}^{i}, \tau_{2}^{i}, \ldots, \tau_{m(i)}^{i}$, and death (censoring) occurred at age $T_{i}$ is

$$
\begin{aligned}
& \widehat{L}_{i}\left(\theta^{i}\left(\tau_{1}^{i}\right), \theta^{i}\left(\tau_{2}^{i}\right), \ldots, \theta^{i}\left(\tau_{m(i)}^{i}\right), T_{i}\right) \\
& =p\left(\theta^{i}\left(t_{0}\right)\right) \prod_{p=1}^{m(i)} \widehat{\lambda}_{\theta^{i}\left(\tau_{p}^{i}-\right), \theta^{i}\left(\tau_{p}^{i}\right)}\left(\tau_{p}^{i}\right) \widehat{\mu}_{\theta^{i}\left(\tau_{m(i)}^{i}\right)}\left(T^{i}\right)^{\delta_{i}} \\
& \quad \times \exp \left(-\int_{t_{0}}^{T_{i}}\left(\sum_{k=1, k \neq \theta^{i}(t-)}^{M} \widehat{\lambda}_{\theta_{t-,}^{i}}(t)+\widehat{\mu}_{\theta_{t-}^{i}}(t)\right) d t\right) .
\end{aligned}
$$

Here $p\left(\theta^{i}\left(t_{0}\right)\right)$ is the initial distribution of the health status and $\theta\left(\tau_{1}^{i}-\right)=\theta\left(t_{0}\right)$ by definition.

Because health transitions are observed, the equations for the first two moments are simplified (Appendix B):

$$
\begin{aligned}
& \frac{d \widehat{m}_{j}(t)}{d t}=-a_{j}(t) \widehat{f}_{1 j}(t)+\sum_{k=1, k \neq j}^{M} \widehat{\gamma}_{j}(t) \Lambda_{j k}(t) \widehat{g}_{j}(t)+2 \widehat{\gamma}_{j}(t) Q_{j}(t) \widehat{f}_{j}(t), \\
& \frac{d \widehat{\gamma}_{j}(t)}{d t}= a_{j}(t) \widehat{\gamma}_{j}(t)+\widehat{\gamma}_{j}(t) a_{j}^{*}(t)+B_{j}(t) \\
&-\sum_{k=1, k \neq j}^{M} 2 \widehat{\gamma}_{j}(t) \Lambda_{j k}(t) \widehat{\gamma}_{j}(t)-2 \widehat{\gamma}_{j}(t) Q_{j}(t) \widehat{\gamma}_{j}(t) .
\end{aligned}
$$

Here $\widehat{f}_{j}(t)=f_{j}(t)-\widehat{m}_{j}(t), \widehat{g}_{j}(t)=g_{j}(t)-\widehat{m}_{j}(t), \widehat{f}_{1 j}(t)=f_{1 j}(t)-\widehat{m}_{j}(t)$. The index $j$ indicates the dependence of these moments on the value of the process $\theta_{t}$ at time $t$. These moments depend on the entire trajectory of $\theta_{t}$ over the interval $\left[t_{0}, t\right)$. Eq. (44) and (45) must be solved over 
the intervals $\left[\tau_{1}, \tau_{2}\right),\left[\tau_{2}, \tau_{3}\right), \ldots,\left[\tau_{m}, T\right)$, between subsequent jumps of the process $\theta_{t}$. When $\theta\left(\tau_{p^{-}}\right)=k$ and $\theta^{i}\left(\tau_{p}\right)=j$, and $\lambda_{k j}\left(Y_{t}, t\right)$ is described by Eq. (14), we have for the initial values $\widehat{m}_{j}\left(\tau_{p}\right)$ and $\widehat{\gamma}_{j}\left(\tau_{p}\right)$ (Appendix C):

$$
\begin{gathered}
\widehat{m}_{j}\left(\tau_{p}\right)=\widehat{m}_{k}\left(\tau_{p}-\right)-\frac{2 \widehat{\gamma}_{k}\left(\tau_{p}-\right) \Lambda_{k j}\left(\tau_{p}\right) \widehat{g}_{k}\left(\tau_{p}-\right)}{\widehat{\lambda}_{k j}\left(\tau_{p}-\right)}, \\
\widehat{\gamma}_{j}\left(\tau_{p}\right)=\widehat{\gamma}_{k}\left(\tau_{p}-\right)+\frac{2 \widehat{\gamma}_{k}\left(\tau_{p}-\right) \Lambda_{k j}\left(\tau_{p}\right) \widehat{\gamma}_{k}\left(\tau_{p}-\right)}{\widehat{\lambda}_{k j}\left(\tau_{p}-\right)}
\end{gathered}
$$

with

$$
\widehat{g}_{k}\left(\tau_{p}-\right)=g_{k}\left(\tau_{p}\right)-\widehat{m}_{k}\left(\tau_{p}-\right)
$$

and

$$
\widehat{\lambda}_{k j}(t)=\lambda_{0 k j}(t)+\left(\widehat{m}_{k}(t)-g_{k}(t)\right)^{*} \Lambda_{k j}(t)\left(\widehat{m}_{k}(t)-g_{k}(t)\right)+\operatorname{Tr}\left(\Lambda_{k j}(t) \widehat{\gamma}_{k}(t)\right) .
$$

\subsection{Plan 3: Discrete-Time Observations of Physiological State and Health Transitions}

In Plan 3 the data from Plans 1 and 2 are combined: the physiological state is measured repeatedly in a sequence of discrete times, and all health transitions are observed. In this case the changes in information about the $i$-th individual take place at times when the process $Y_{t}$ is measured or when the process $\theta_{t}$ jumps. The last observation of the health transition is $\tau_{m(i)}^{i}$ and $t_{n(i)}^{i}$ is the last measurement of physiological state of individual $i, \quad \tau_{m(i)}^{i}<T_{i}$ and $t_{n(i)}^{i}<T_{i}$. Each individual is characterized by the ordered sequence of ages at which different measurements took place. For example, the sequence $\left(t_{1}, t_{2}\right.$, $\left.\tau_{1}, t_{3}, \tau_{2}, \tau_{3}, t_{4}, \ldots, \tau_{m}\right)$ indicates that the first health transition occurred between the second and the third measurements of the physiological state, that the second and third health transitions occurred between the third and fourth physiological measurements, and so on. $P\left(\tau_{k}=t_{r}\right)=0$ for any $k$ and $r$.

The conditional probability density function

$$
\hat{f}(y, t)=\frac{\partial}{\partial y} P\left(Y_{t} \leq y \mid \widetilde{Y}_{0}^{t}, \theta_{0}^{t}, T>t\right)
$$

of $Y_{t}$ given observations $\widetilde{Y}_{0}^{t}, \theta_{0}^{t}$ and $T>t$ satisfies Eq. (39) with $\widehat{\lambda}_{\theta_{t-}, k}(t)$ and $\widehat{\mu}_{\theta_{t-}}(t)$ replaced by $\hat{\lambda}_{\theta_{t-}, k}(t)$ and $\hat{\mu}_{\theta_{t-}}(t)$, where 


$$
\hat{\lambda}_{\theta_{t-}, k}(t)=E\left(\lambda_{\theta_{t-}, k}\left(Y_{t}, t\right) \mid \widetilde{Y}_{0}^{t}, \theta_{0}^{t-}, T>t\right)
$$

and

$$
\hat{\mu}_{\theta_{t-}}(t)=E\left(\mu_{\theta_{t-}}\left(Y_{t}, t\right) \mid \widetilde{Y}_{0}^{t}, \theta_{0}^{t-}, T>t\right),
$$

over each interval combining and ordering $t_{1}, t_{2}, \ldots, t_{n}$ and $\tau_{1}, \tau_{2}, \ldots$, $\tau_{m}$. If the interval starts with $t_{k}$, then modified Eq. (39) for $\hat{f}(y, t)$ must be used with initial condition $\hat{f}\left(y_{t_{k}}, t_{k}\right)=\delta\left(y-y_{t_{k}}\right)$. If the interval starts with $\tau_{p}$ then Eq. (39) must be used with initial conditions:

$$
\hat{f}\left(y, \tau_{p}\right)=\hat{f}\left(y, \tau_{p}-\right) \frac{\lambda_{\theta\left(\tau_{p}-\right), \theta\left(\tau_{p}\right)}\left(y, \tau_{p}-\right)}{\hat{\lambda}_{\theta\left(\tau_{p}-\right), \theta\left(\tau_{p}\right)}\left(\tau_{p}-\right)},
$$

where

$$
\hat{\lambda}_{\theta\left(\tau_{p}-\right), \theta\left(\tau_{p}\right)}\left(\tau_{p}\right)=\left.E\left(\lambda_{\theta\left(\tau_{p}-\right), x}\left(Y_{\tau_{p}}, \tau_{p}\right) \mid \widetilde{Y}_{0}^{\tau_{p}}, \theta_{0}^{\tau_{p}-}, T>\tau_{p}\right)\right|_{x=\theta\left(\tau_{p}\right)} .
$$

An example of likelihood function for the $i$-th individual with observations occurring at times $t_{1}^{i}, t_{2}^{i}, \tau_{1}^{i}, t_{3}^{i}, \tau_{2}^{i}, \tau_{3}^{i}, \ldots, \tau_{m(i)}^{i}, t_{n(i)}^{i}, T^{i}$ in case of Plan 3 is

$$
\begin{aligned}
& \widehat{L_{i}}\left(y_{t_{1}^{i}}^{i}, y_{t_{2}^{i}}^{i}, \ldots, y_{t_{n(i)}^{i}}^{i}, \theta^{i}\left(\tau_{1}^{i}\right), \theta^{i}\left(\tau_{2}^{i}\right), \ldots, \theta^{i}\left(\tau_{m(i)}^{i}\right), T_{i}\right) \\
& =\hat{f}\left(y_{t_{1}^{i}}, t_{1}^{i}-\right) \hat{f}\left(y_{t_{2}^{i}}, t_{2}^{i}-\right) \hat{f}\left(y_{\tau_{1}^{i}}, \tau_{1}^{i}-\right) \hat{f}\left(y_{t_{3}^{i}}, t_{3}^{i}-\right) \hat{f}\left(y_{\tau_{2}^{i}}, \tau_{2}^{i}-\right) \\
& \quad \times \hat{f}\left(y_{\tau_{3}^{i}}, \tau_{3}^{i}-\right) \ldots \hat{f}\left(y_{s^{i}}, s^{i}-\right) p\left(\theta^{i}\left(t_{0}^{i}\right)\right) \prod_{p=1}^{m(i)} \hat{\lambda}_{\theta^{i}\left(\tau_{p}^{i}-\right), \theta^{i}\left(\tau_{p}^{i}\right)}\left(\tau_{p}^{i}\right) \hat{\mu}_{\theta^{i}\left(T_{i}\right)}\left(T^{i}\right)^{\delta_{i}} \\
& \quad \times \exp \left(-\int_{t_{0}^{i}}^{T_{i}}\left(\sum_{k=1, k \neq \theta^{i}(t-)}^{M} \hat{\lambda}_{\theta_{t-}^{i}, k}(t)+\hat{\mu}_{\theta_{t-}^{i}}(t)\right) d t\right) .
\end{aligned}
$$

Here $s^{i}=\max \left\{t_{n(i)}^{i}, \tau_{m(i)}^{i}\right\}$, and $M$ is the total number of states of the process $\theta_{t} . \hat{f}\left(y, t_{k}^{i}-\right)=\frac{\partial}{\partial y} P\left(Y_{t_{k}^{i}} \leq y \mid \widetilde{Y}_{0}^{t_{k}^{i}-}, \theta_{0}^{t_{k}^{i}-}, T>t_{k}^{i}\right)$ is the solution of Eq. (39) either over the interval $\left[\tau_{p}^{i}, t_{k}^{i}\right)$, or over the interval $\left[t_{k-1}^{i}, t_{k}^{i}\right)$, assuming that these intervals contain no other observations; $\hat{f}\left(y, \tau_{k}^{i}-\right)=\frac{\partial}{\partial y} P\left(Y_{\tau_{k}^{i}} \leq y \mid \widetilde{Y}_{0}^{\tau_{k}^{i}-}, \theta_{0}^{\tau_{k}^{i}-}, T>\tau_{k}^{i}\right)$ is the solution of Eq. (39) either over the interval $\left[\tau_{k-1}^{i}, \tau_{k}^{i}\right)$, or over the interval $\left[t_{p}^{i}, \tau_{k}^{i}\right)$, assuming that these intervals contain no other observation.

The first two moments of the conditional probability density function $\hat{f}(y, t)$ defined in Eq. (50) are $\hat{m}(t)=E\left(Y_{t} \mid \widetilde{Y}_{0}^{t}, \theta_{0}^{t}, T>t\right)$ and $\hat{\gamma}(t)=E\left(\left(Y_{t}-\hat{m}(t)\right)\left(Y_{t}-\hat{m}(t)\right)^{*} \mid \widetilde{Y}_{0}^{t}, \theta_{0}^{t}, T>t\right)$. These moments satisfy 
Eq. (44) and (45) over each interval combining and ordering $t_{1}, t_{2}, \ldots$, $t_{n}$ and $\tau_{1}, \tau_{2}, \ldots, \tau_{m}$.

If the interval starts with $t_{k}$, then Eq. (44) for $\hat{m}(t)$ starts with initial condition $\hat{m}\left(t_{k}\right)=y_{t_{k}}$ and for $\hat{\gamma}(t)$ with condition $\hat{\gamma}\left(t_{k}\right)=0$. If the interval starts with $\tau_{p}$ then Eq. (44) starts with initial condition given by Eq. (46) and (47) with $\widehat{m}\left(\tau_{p^{-}}\right)$and $\widehat{\gamma}\left(\tau_{p^{-}}\right)$replaced by $\hat{m}\left(\tau_{p}-\right)$ and $\hat{\gamma}\left(\tau_{p}-\right)$.

The likelihood function of the data for the $i$-th individual in the case of Plan 3 is

$$
\begin{aligned}
& \widehat{L}_{i}^{G}\left(y_{t_{1}^{i}}^{i}, y_{t_{2}^{i}}^{i}, \ldots, y_{t_{n(i)}^{i}}^{i}, \theta^{i}\left(\tau_{1}^{i}\right), \theta^{i}\left(\tau_{2}^{i}\right), \ldots, \theta^{i}\left(\tau_{m(i)}^{i}\right), T_{i}\right) \\
& =p\left(\theta^{i}\left(t_{0}^{i}\right)\right) \prod_{j=1}^{n(i)} \chi\left(y_{u_{j}^{i}}^{i}, u_{j}^{i}\right) p\left(\theta^{i}\left(t_{0}^{i}\right)\right) \prod_{p=1}^{m(i)} \hat{\lambda}_{\theta^{i}\left(\tau_{p}^{i}-\right), \theta^{i}\left(\tau_{p}^{i}\right)}\left(\tau_{p}^{i}\right) \hat{\mu}_{\theta^{i}\left(T_{i}\right)}\left(T^{i}\right)^{\delta_{i}} \\
& \quad \times \exp \left(-\int_{t_{0}^{i}}^{T_{i}}\left(\sum_{k=1, k \neq \theta^{i}(t-)}^{M} \hat{\lambda}_{\theta_{t-}^{i}, k}(t)+\hat{\mu}_{\theta_{t-}^{i}}(t)\right) d t\right),
\end{aligned}
$$

where $\chi(y, t)$ is similar to Eq. (32) with $\tilde{\gamma}_{k}^{i}(t)$ and $\tilde{m}_{k}^{i}(t)$ replaced by $\hat{\gamma}^{i}(t)$ and $\hat{m}^{i}(t)$, respectively, $u_{k}^{i}, k=1,2, \ldots, n(i)+m(i)$ is the element of an ordered sequence combined from $t_{1}^{i}, t_{2}^{i}, \ldots, t_{n(i)}^{i}$ and $\tau_{1}^{i}, \tau_{2}^{i}, \ldots, \tau_{m(i)}^{i}$. If the interval starts with $t_{k}^{i}$ then Eq. (44) and (45) start with initial conditions $m_{t^{i}}=y_{t_{b}^{i}}, \gamma_{t^{i}}=0$. If the interval starts with $\tau_{p}^{i}$ then Eq. (44) and (45) start with initial conditions given by Eq. (46) and (47). The mortality rate is

$$
\hat{\mu}(t)=\mu_{0}(t)+\left(\hat{m}(t)-f_{j}(t)\right)^{*} Q_{j}(t)\left(\hat{m}(t)-f_{j}(t)\right)+\operatorname{Tr}\left(Q_{j}(t) \hat{\gamma}(t)\right),
$$

and the transition intensities $\hat{\lambda}_{k j}(t)$ are

$$
\hat{\lambda}_{k j}(t)=\lambda_{0 k j}(t)+\left(\hat{m}(t)-g_{k}(t)\right)^{*} \Lambda_{k j}(t)\left(\hat{m}(t)-g_{k}(t)\right)+\operatorname{Tr}\left(\Lambda_{k j}(t) \hat{\gamma}(t)\right) .
$$

The likelihood function (56) can be used for combining data collected using Plans 1 and 2 for the same individuals.

\section{SIMULATION}

We simulated data keeping in mind the design of the Framingham Heart Study (FHS) (Dawber, 1980). We assumed that each individual is characterized by a continuously changing physiological index $Y_{t}$, 
whose dynamics is described by the stochastic differential Eq. (16), and be either in the healthy or unhealthy state. For each individual, we simulated both one-year survival and a disease onset using the quadratic hazard of Eq. (15) and (14), respectively. We considered healthy (marked by letter ' $\mathrm{H}$ ') and diseased individuals (marked by letter ' $D$ '). We assumed: i) time independence of parameters describing the dynamics in both states: $a_{H}, a_{D}, b_{H}, b_{D}, f_{1 H}$, and $f_{1 D}$, ii) the Gompertz function for $\mu_{0 i}(t)=\mu_{0 i} \exp \left(\alpha_{i} t\right), \quad i=H, \quad D$, and for $\lambda_{0 H D}(t)=\lambda_{O H D} \exp \left(\alpha_{H D} t\right)$, and iii) the time independence of other parameters describing the transition probabilities: $Q_{H}, Q_{D}, f_{H}, f_{D}, \Lambda_{H D}$, and $g_{H}$. Parameters for simulations result from the one-state stochastic process for healthy and unhealthy subcohorts in the Framingham Heart Study (FHS). The cohort of healthy individuals with data on mortality and incidence of chronic disease provided dynamic and mortality parameters for the healthy state. The cohort of individuals considered after an onset of a chronic disease allowed us to evaluate dynamic and mortality parameters for the unhealthy state. We used: $a_{H}=-0.05, \quad a_{D}=-0.03, \quad b_{H}^{2}=10, \quad b_{D}^{2}=15, \quad f_{1 H}=80, \quad f_{1 D}=85$, $\mu_{0 H}=0.00002, \quad \alpha_{H}=0.08, \quad \mu_{0 D}=0.002, \quad \alpha_{D}=0.045, \quad Q_{H}=0.00001$, $Q_{D}=0.00007, \Lambda_{H D}=0.00005, \lambda_{0 H D}=0.00005, \alpha_{H D}=0.065, f_{H}=80$, $f_{D}=80$, and $g_{H}=72$. Here $f_{1 H}, f_{1 D}, f_{H}, f_{D}, g_{H}, b_{H}$, and $b_{D}$ are in the units of the used covariate (e.g., mmHg for blood pressure), $a_{H}$ and $a_{D}$ are dimensionless, $\mu_{0 H}, \alpha_{H}, \mu_{0 D}, \alpha_{D}, \lambda_{0 H D}$, and $\alpha_{H D}$ are in year ${ }^{-1}$, and $Q_{H}, Q_{D}$, and $\Lambda_{H D}$ are in year ${ }^{-1}$ multiplied by the reciprocal of the covariate unit squared.

We simulated 10 cohorts using these parameters. The starting age for individuals in these cohorts was 50 years, and $25 \%$ of individuals were assigned to the unhealthy state. Starting values for diastolic blood pressure were $80 \mathrm{mmHg}$ for healthy and $85 \mathrm{mmHg}$ for unhealthy individuals. Death and transition to unhealthy states were simulated for each month. The vital status, the health state, and the covariate value were recorded annually. The characteristics of simulated cohorts were compared with the theoretical two-stage model. The conditional hazard, or mortality rate, given health status and unconditional transition intensity functions of Eq. (17) and (18) are specified as:

$$
\begin{array}{r}
\bar{\mu}_{H}(t)=\mu_{0 H}(t)+\left(m_{H}(t)-f_{H}(t)\right)^{2} Q_{H}(t)+Q_{H}(t) \gamma_{H}(t), \\
\bar{\mu}_{D}(t)=\mu_{0 D}(t)+\left(m_{D}(t)-f_{D}(t)\right)^{2} Q_{D}(t)+Q_{D}(t) \gamma_{D}(t), \\
\bar{\lambda}_{H D}(t)=\lambda_{0 H D}(t)+\left(m_{H}(t)-g_{H}(t)\right)^{2} \Lambda_{H D}(t)+\Lambda_{H D}(t) \gamma_{H}(t) .
\end{array}
$$


The differential Eq. (21) and (22) for the conditional moments become:

$$
\begin{aligned}
& \frac{d m_{H}(t)}{d t}=2 \gamma_{H}(t) \Lambda_{H D}(t) \breve{g}_{H}(t)-a_{H}(t) \breve{f}_{1 H}(t)+2 \gamma_{H}(t) Q_{H}(t) \breve{f}_{H}(t), \\
& \frac{d m_{D}(t)}{d t}=\frac{\pi_{H}(t)}{\pi_{D}(t)}\left(m_{H D}(t) \bar{\lambda}_{H D}-2 \gamma_{H}(t) \Lambda_{H D} \breve{g}_{H}(t)\right) \\
& -a_{D}(t) \breve{f}_{1 D}(t)+2 \gamma_{D}(t) Q_{D}(t) \breve{f}_{D}(t) \\
& \frac{d \gamma_{H}(t)}{d t}=-2 \gamma_{H}^{2}(t) \Lambda_{H D}(t)+2 a_{H}(t) \gamma_{H}(t)+b_{H}^{2}(t)-2 \gamma_{H}^{2}(t) Q_{H}(t), \\
& \frac{d \gamma_{D}(t)}{d t}=\frac{\pi_{H}(t)}{\pi_{D}(t)}\left(\left(\gamma_{H}(t)-\gamma_{D}(t)+m_{H D}^{2}(t)\right) \bar{\lambda}_{H D}(t)\right. \\
& \left.+2\left(\gamma_{H}^{2}(t) \Lambda_{H D}(t)-2 \gamma_{H}(t) \Lambda_{H D}(t) \breve{g}_{H}(t) m_{H D}(t)\right)\right) \\
& +2 a_{D}(t) \gamma_{D}(t)+b_{D}^{2}(t)-2 \gamma_{D}^{2}(t) Q_{D}(t),
\end{aligned}
$$

and the equations for the conditional probabilities of being in healthy and unhealthy states are

$$
\begin{gathered}
\frac{d \pi_{H}(t)}{d t}=-\pi_{H}(t) \bar{\lambda}_{H D}(t)+\pi_{H}(t)\left(\pi_{H}(t) \bar{\mu}_{H}(t)+\pi_{D}(t) \bar{\mu}_{D}(t)-\bar{\mu}_{H}(t)\right), \\
\frac{d \pi_{D}(t)}{d t}=\pi_{H}(t) \bar{\lambda}_{H D}(t)+\pi_{D}(t)\left(\pi_{H}(t) \bar{\mu}_{H}(t)+\pi_{D}(t) \bar{\mu}_{D}(t)-\bar{\mu}_{D}(t)\right) .
\end{gathered}
$$

Here $m_{H D}(t)=m_{H}(t)-m_{D}(t)$.

Figure 1 shows the theoretical curves, corresponding to Eq. (59) to (67), calculated with the parameters used in the simulation, and the age patterns of respective characteristics in simulated cohorts obtained by averaging individual trajectories of 5,000 individuals. The comparison indicates that the theoretical curves correspond to simulated data.

For each simulated cohort, the model parameters were estimated from Plans 1, 2, and 3, and we compared the estimates with their original values used for simulation. All 18 parameters were subject for estimation in Plan 3. Two different sets of 12 parameters were chosen for estimation in Plans 1 and 2.

We first assumed that the data are collected according to Plan 1 and the estimation resulted from the likelihood of Eq. (31). Plan 1 is insufficient for evaluating all parameters. Therefore, twelve parameters 

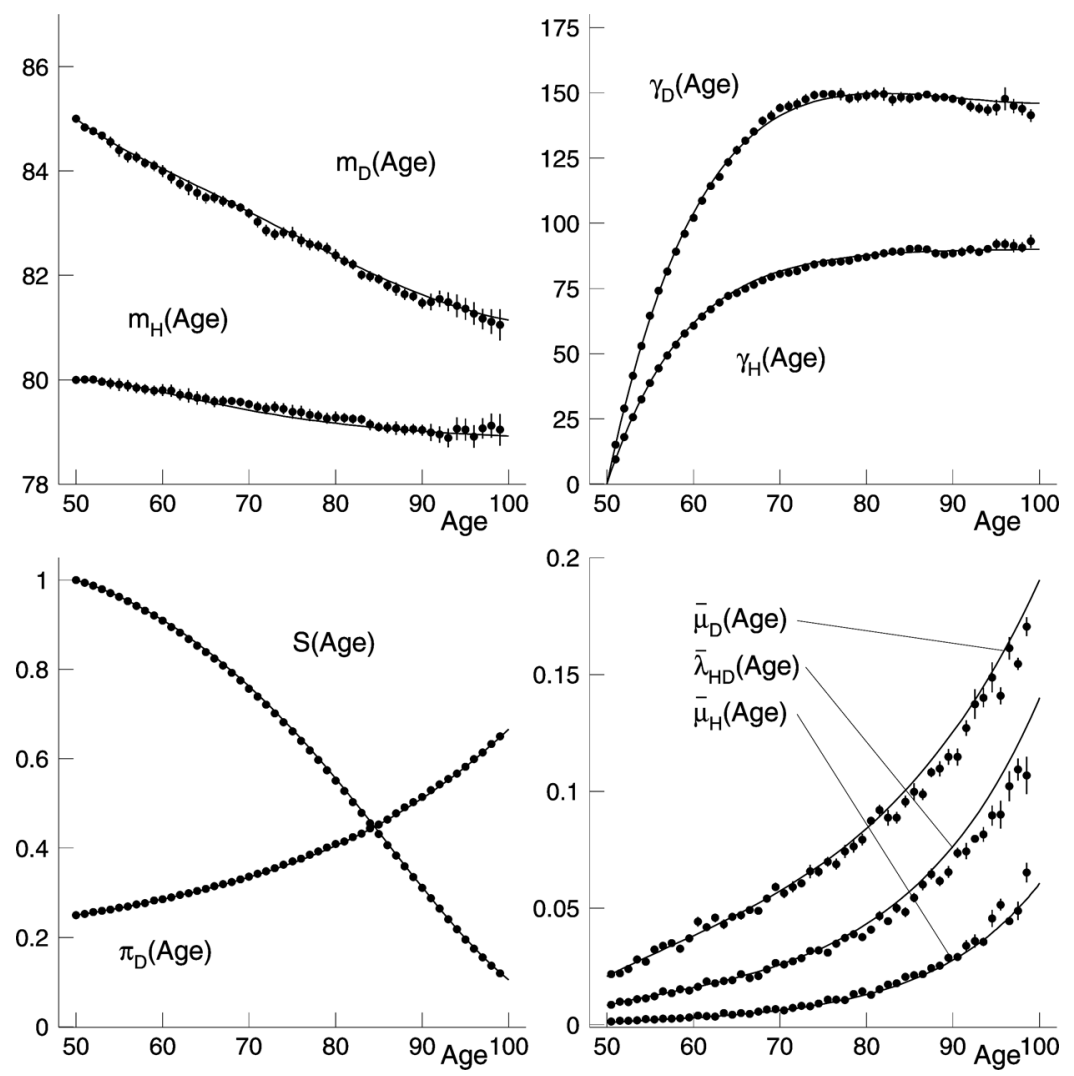

FIGURE 1 Age patterns of characteristics of simulated cohorts: i) defined by the true parameters used for simulation (represented by solid lines) and ii) means of empiric estimates of respective characteristics over 10 simulated cohorts represented by dots with error bars. First moments for healthy and disease states, $m_{H}(t)$ and $m_{D}(t)$ (left-upper panel); second central moments for healthy and disease states, $\gamma_{H}(t)$ and $\gamma_{D}(t)$ (right-upper panel); survival function and prevalence of unhealthy individuals (left-lower panel); mortality rates from healthy and unhealthy states and transition rate from healthy to unhealthy states (right-lower panel).

presented in Table 1 were estimated while the others were fixed and equal to their input values. The estimated parameters are responsible for the shape of trajectories of the covariate in healthy and unhealthy states and for mortality from these states. Table 1 shows that parameters of changes in physiological indices are estimated with a good accuracy. 
TABLE 1 The Results of the Simulation Experiment for Plans 1, 2, and 3 with 10 Datasets ([C] Denotes Dimensionality of a Covariate: Millimeters of Mercury (mmHg))

\begin{tabular}{|c|c|c|c|c|c|c|c|c|}
\hline \multirow{2}{*}{$\begin{array}{l}\text { Model } \\
\text { parameters }\end{array}$} & \multirow[b]{2}{*}{ Units } & \multirow[b]{2}{*}{ true } & \multicolumn{2}{|c|}{ Plan 1} & \multicolumn{2}{|c|}{ Plan 2} & \multicolumn{2}{|c|}{ Plan 3} \\
\hline & & & Mean & $\mathrm{SD}$ & Mean & $\mathrm{SD}$ & Mean & $\mathrm{SD}$ \\
\hline$a_{H}$ & & -0.05 & -0.049 & 0.002 & & & -0.051 & 0.002 \\
\hline$a_{D}$ & & -0.03 & -0.030 & 0.002 & & & -0.03 & 0.002 \\
\hline$b_{H}$ & {$[\mathrm{C}]$} & 10 & & & & & 9.97 & 0.06 \\
\hline$b_{D}$ & {$[\mathrm{C}]$} & 15 & & & & & 15 & 0.07 \\
\hline$f_{1 H}$ & {$[\mathrm{C}]$} & 80 & 80.5 & 0.4 & & & 80.1 & 0.25 \\
\hline$f_{1 D}$ & {$[\mathrm{C}]$} & 85 & 84.2 & 0.6 & & & 84.81 & 0.55 \\
\hline$\mu_{0 H} \times 10^{5}$ & Year $^{-1}$ & 2 & 0.36 & 0.16 & 1.83 & 1.03 & 1.96 & 0.33 \\
\hline$\alpha_{H}$ & Year $^{-1}$ & 0.08 & 0.099 & 0.008 & 0.082 & 0.008 & 0.082 & 0.002 \\
\hline$Q_{H} \times 10^{5}$ & $\operatorname{Year}^{-1}[\mathrm{C}]^{-2}$ & 1 & 3 & 0.77 & 1.29 & 0.77 & 1.23 & 0.21 \\
\hline$\mu_{0 D} \times 10^{5}$ & Year $^{-1}$ & 200 & 187.9 & 49.6 & 135.8 & 48.8 & 201.3 & 24.3 \\
\hline$\alpha_{D}$ & Year $^{-1}$ & 0.045 & 0.046 & 0.003 & 0.049 & 0.003 & 0.045 & 0.001 \\
\hline$Q_{D} \times 10^{5}$ & $\operatorname{Year}^{-1}[\mathrm{C}]^{-2}$ & 7 & 6.46 & 0.66 & 11.02 & 3.39 & 7.14 & 0.5 \\
\hline$\lambda_{O H D} \times 10^{5}$ & Year $^{-1}$ & 20 & & & 28.56 & 19.35 & 23.5 & 4.77 \\
\hline$\alpha_{H D}$ & Year $^{-1}$ & 0.065 & & & 0.064 & 0.008 & 0.063 & 0.002 \\
\hline$\Lambda_{H D} \times 10^{5}$ & $\operatorname{Year}^{-1}[\mathrm{C}]^{-2}$ & 5 & & & 4.52 & 4.00 & 4.66 & 0.62 \\
\hline$f_{H}$ & {$[\mathrm{C}]$} & 80 & 75.9 & 2.5 & 79.52 & 4.63 & 79.32 & 1.34 \\
\hline$f_{D}$ & {$[\mathrm{C}]$} & 80 & 80.2 & 0.63 & 79.62 & 3.42 & 80.04 & 0.96 \\
\hline$g_{H}$ & {$[\mathrm{C}]$} & 72 & & & 73.47 & 34.21 & 71.7 & 1.02 \\
\hline
\end{tabular}

Then we assumed that data are collected according to Plan 2: only ages at onset and death are observed. Twelve parameters chosen for estimation describe the transition probabilities. Table 1 shows that the standard deviation of obtained estimates is larger. The parameters of transition rates are estimated accurately if the parameters describing physiology are known. This suggests that all parameters might be estimated properly in Plan 3.

When data are collected according to Plan 3 the likelihood of Eq. (56) is used. Some specifications of the likelihood to simplify the programming of optimization procedure using SAS Proc NLP are given in Appendix D. All 18 parameters were estimated for each cohort. Means and standard deviations of the estimated parameters obtained by averaging the ten simulated cohorts are given in Table 1. Figure 2 shows that the accuracy of parameter estimation is good. It presents age trajectories for simulated cohorts calculated with the estimated parameter values in comparison with the trajectories obtained from the original values.

A bias observed between empirical and reconstructed parameters may arise because two subsequent transitions occur over a fixed time interval (e.g., a transition from healthy to unhealthy and a transition to death). If the time between the two transitions is small enough, the 

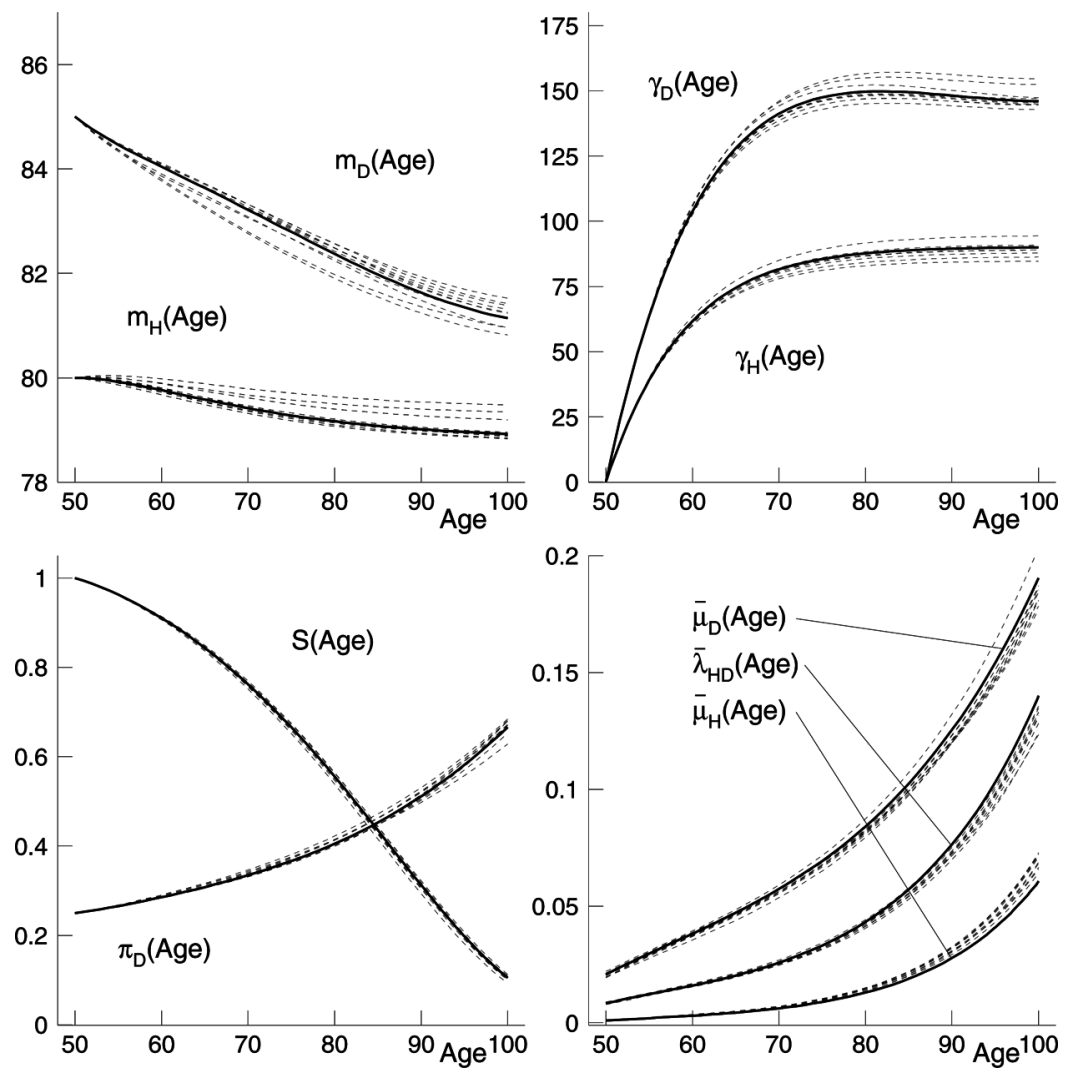

FIGURE 2 Age patterns of physiological and life history characteristics calculated from theoretical model (solid lines), and using estimates of parameters obtained by maximization of the likelihood for Plan 3 for ten simulated cohorts with 5,000 individuals in each (dashed lines). The same characteristics are presented in panels as in Figure 1.

death transitions can be interpreted in Plan 3 as a single transition from healthy to death.

\section{CONCLUSION}

To evaluate the connection between age trajectories of physiological state, changes in health status, and survival in humans, we investigated the joint evolution of a system of indices representing such changes and indicators of human health and survival. Such joint evolution is represented in a number of data sets collected in longitudinal studies of aging, health, and longevity. A good example is the 
Framingham Heart Study (Dawber, 1980), in which data on the original cohort contain the results of biennial examinations of physiological state, biological indicators, and ages at onset of a number of chronic conditions, such as cardiovascular disease, cancer, and type 2 Diabetes Mellitus, performed during over 60 years. The progress in this field depends on the possibility of joint analysis of data describing the processes of aging related changes and disease development.

We showed that developing mortality models for studying health and aging requires incorporating knowledge about aging-related changes into the model. Connecting physiological state with health status and mortality makes model parameters interpretable in terms of properties of respective aging-related processes. A future step will be to investigate the connection among aging, health, and fecundity (Yashin et al., 1998) or aging processes in related individuals (Yashin and Iachine, 1995).

\section{REFERENCES}

Akushevich, I., Kulminski, A., and Manton, K. (2005). Life tables with covariates: Dynamic model for nonlinear analysis of longitudinal data. Mathematical Population Studies, 12: 51-80.

Arbeev, K.G., Akushevich, I., Kulminski, A., et al. (2009). Genetic model for longitudinal studies of aging, health, and longevity and its potential application to incomplete data. Journal of Theoretical Biology, 258: 103-111.

Bagdonavicius, V. and Nikulin, M. (2009). Statistical models to analyze failure, wear, fatigue, and degradation data with explanatory variables. Communications in Statistics-Theory and Methods, 38(16-17): 3031-3047.

Dawber, T.R. (1980). The Framingham Study: The Epidemiology of Atherosclerotic Disease. Cambridge, MA: Harvard University Press.

Sacher, G. and Trucco, E. (1962). The stochastic theory of mortality. Annals of the New York Academy of Sciences, 96: 985-1007.

Strehler, B.L. and Mildvan, A.S. (1960). General theory of mortality and aging. Science, 132(3418): 14-21.

Woodbury, M.A. and Manton, K.G. (1977). A random walk model of human mortality and aging. Theoretical Population Biology, 11: 37-48.

Yashin, A.I. (1980). Conditional Gaussian estimation of dynamic systems under jumping observations. Automation and Remote Control, 5: 123-129.

Yashin, A.I. (1985). Dynamics in survival analysis: Conditional Gaussian property versus Cameron-Martin formula. In N.V. Krylov, R.Sh. Liptser, and A.A. Novikov (Eds.), Statistics and Control of Stochastic Processes. New York, NY: Springer, pp. 446-485.

Yashin, A.I. and Manton, K.G. (1997). Effects of unobserved and partially observed covariate processes on system failure: A review of models and estimation strategies. Statistical Science, 12: 20-34.

Yashin, A.I., Arbeev, K.G., Akushevich, I., et al. (2007). Stochastic model for analysis of longitudinal data on aging and mortality. Mathematical Biosciences, 208: 538-551.

Yashin, A.I., Arbeev, K.G., Akushevich, I., et al. (2008). Model of hidden heterogeneity in longitudinal data. Theoretical Population Biology, 73: 1-10. 
Yashin, A.I., Manton, K.G., Woodbury, M.A., and Stallard, E. (1995). The effects of health histories on stochastic process models of aging and mortality. Journal of Mathematical Biology, 34: 1-16.

Yashin, A.I. and Iachine, I.A. (1995). Survival of related individuals: An extension of some fundamental results of heterogeneity analysis. Mathematical Population Studies, 5(4): 321-339.

Yashin, A.I., Iachine, I.A., Andreev, K.F., and Larsen, U. (1998). Multistate models of postpartum infecundity, fecundability and sterility by age and parity: Methodological issues. Mathematical Population Studies, 7(1): 51-78.

Yashin, A.I., Iachine, I.A., and Begun, A.S. (2000). Mortality modeling: A review. Mathematical Population Studies, 8(4): 305-332.

\section{APPENDIX A}

There are two types of indices: i) vector index of $y$ denoted by Greek symbols and ii) index counting discrete states denoted by Latin symbols. Introduce $x_{j}^{\alpha}=y^{\alpha}-m_{j}^{\alpha}(t), m_{i j}(t)=m_{i}(t)-m_{j}(t)$ and define "hat" variables as $\check{f}_{j}^{\alpha}(t)=f_{j}^{\alpha}(t)-m_{j}^{\alpha}(t), \check{f}_{1 j}^{\alpha}(t)=f_{1 j}^{\alpha}(t)-m_{j}^{\alpha}(t), \breve{g}_{i}^{\alpha}(t)=$ $g_{i}^{\alpha}(t)-m_{i}^{\alpha}(t)$. Rewrite Eq. (13) for $f(y \mid j, t)$ :

$$
\begin{aligned}
\frac{\partial f(y \mid j, t)}{\partial t}= & \sum_{i=1}^{N} \frac{\pi_{i}(t)}{\pi_{j}(t)}\left(\lambda_{i j}(y, t) f(y \mid i, t)-\bar{\lambda}_{i j}(t) f(y \mid j, t)\right)-\frac{\partial A_{j}(y, t) f(y \mid j, t)}{\partial y} \\
& +\frac{B_{j}(t)}{2} \frac{\partial^{2} f(y \mid j, t)}{\partial y^{2}}+\left(\bar{\mu}_{j}(t)-\mu_{j}(y, t)\right) f(y \mid j, t) .
\end{aligned}
$$

The assumptions are

$$
\begin{aligned}
\mu_{j}(y, t) & =\mu_{0 j}(t)+\sum_{\alpha \beta}\left(y^{\alpha}-f_{j}^{\alpha}(t)\right)\left(y^{\beta}-f_{j}^{\beta}(t)\right) Q_{j}^{\alpha \beta}(t) \\
& =\mu_{0 j}(t)+\sum_{\alpha \beta}\left(x_{j}^{\alpha}-\check{f}_{j}^{\alpha}(t)\right)\left(x_{j}^{\beta}-\check{f}_{j}^{\beta}(t)\right) Q_{j}^{\alpha \beta}(t), \\
\lambda_{i j}(y, t) & =\lambda_{0 i j}(t)+\sum_{\alpha \beta}\left(y^{\alpha}-g_{i}^{\alpha}(t)\right)\left(y^{\beta}-g_{i}^{\beta}(t)\right) \Lambda_{i j}^{\alpha \beta}(t) \\
& =\lambda_{0 i j}(t)+\sum_{\alpha \beta}\left(x_{i}^{\alpha}-\breve{g}_{i}^{\alpha}(t)\right)\left(x_{i}^{\beta}-\check{g}_{i}^{\beta}(t)\right) \Lambda_{i j}^{\alpha \beta}(t) .
\end{aligned}
$$

\section{A.1. First Moment}

$$
\begin{aligned}
\frac{d m_{j}^{\mu}(t)}{d t}= & \sum_{i} \frac{\pi_{i}(t)}{\pi_{j}(t)} \int y^{\mu}\left(\lambda_{i j}(y, t) f(y \mid i, t)-\bar{\lambda}_{i j}(t) f(y \mid j, t)\right) d y \\
& -\int y^{\mu} \frac{\partial A_{j}^{\alpha}(y, t) f(y \mid j, t)}{\partial y^{\alpha}} d y+\frac{B_{j}(t)}{2} \int y^{\mu} \frac{\partial^{2} f(y \mid j, t)}{\partial y^{2}} d y \\
& +\int y^{\mu}\left(\bar{\mu}_{j}(t)-\mu_{j}(y, t)\right) f(y \mid j, t) d y .
\end{aligned}
$$


Here and below all integrals are calculated over the region $R^{K}$. Calculate integrals

$$
\begin{aligned}
& \int y^{\mu}\left(\lambda_{i j}(y, t) f(y \mid i, t)-\bar{\lambda}_{i j} f(y \mid j, t)\right) d y \\
& =\left(m_{i}^{\mu}(t)-m_{j}^{\mu}(t)\right) \bar{\lambda}_{i j}(t)+\int x_{i}^{\mu} \lambda_{i j}(y, t) f(y \mid i, t) d y \\
& =\left(m_{i}^{\mu}(t)-m_{j}^{\mu}(t)\right) \bar{\lambda}_{i j}(t) \\
& +\sum_{\alpha \beta} \Lambda_{i j}^{\alpha \beta}(t) \int x_{i}^{\mu}\left(x_{i}^{\alpha}-\check{g}_{i}^{\alpha}(t)\right)\left(x_{i}^{\beta}-\check{g}_{i}^{\beta}(t)\right) f(y \mid i, t) d y \\
& =\left(m_{i}^{\mu}(t)-m_{j}^{\mu}(t)\right) \bar{\lambda}_{i j}(t)-\sum_{\alpha \beta} \Lambda_{i j}^{\alpha \beta}(t)\left(\gamma_{i}^{\mu \alpha}(t) \check{g}_{i}^{\beta}(t)+\gamma_{i}^{\mu \beta}(t) \check{g}_{i}^{\alpha}(t)\right), \\
& \int y^{\mu} \sum_{\alpha} \frac{\partial\left(A_{j}^{\alpha}(y, t) f(y \mid j, t)\right)}{\partial y^{\alpha}} d y \\
& =\int y^{\mu} \sum_{\alpha \beta} \frac{\partial\left(a_{j}^{\alpha \beta}(t)\left(y^{\beta}-f_{1 j}^{\beta}(t)\right) f(y \mid j, t)\right)}{\partial y^{\alpha}} d y \\
& =\sum_{\beta} \int y^{\mu} \frac{\partial\left(a_{j}^{\mu \beta}(t)\left(y^{\beta}-f_{1 j}^{\beta}(t)\right) f(y \mid j, t)\right)}{\partial y^{\mu}} d y \\
& =-\sum_{\beta} \int a_{j}^{\mu \beta}(t)\left(y^{\beta}-f_{1 j}^{\beta}(t)\right) f(y \mid j, t) d y \\
& =-\sum_{\beta} a_{j}^{\mu \beta}(t)\left(m_{j}^{\beta}(t)-f_{1 j}^{\beta}(t)\right)=\sum_{\beta} a_{j}^{\mu \beta}(t) \check{f}_{1 j}^{\beta}(t), \\
& \int y^{\mu} \frac{\partial^{2} f(y \mid j, t)}{\partial y^{2}} d y=0 \\
& \int y^{\mu}\left(\bar{\mu}_{j}(t)-\mu_{j}(y, t)\right) f(y \mid j, t) d y=-\int x_{j}^{\mu} \mu_{j}(y, t) f(y \mid j, t) d y \\
& =-\sum_{\alpha \beta} Q_{j}^{\alpha \beta}(t) \int x_{j}^{\mu}\left(x_{j}^{\alpha}-\check{f}_{j}^{\alpha}(t)\right)\left(x_{j}^{\beta}-\check{f}_{j}^{\beta}(t)\right) f(y \mid j, t) d y \\
& =\sum_{\alpha \beta} Q_{j}^{\alpha \beta}(t)\left(\gamma_{j}^{\mu \alpha}(t) \check{f}_{j}^{\beta}(t)+\gamma_{j}^{\mu \beta}(t) \check{f}_{j}^{\alpha}(t)\right) .
\end{aligned}
$$


Hence,

$$
\begin{aligned}
\frac{d m_{j}^{\mu}(t)}{d t}= & \sum_{i} \frac{\pi_{i}(t)}{\pi_{j}(t)}\left(\left(m_{i}^{\mu}(t)-m_{j}^{\mu}(t)\right) \bar{\lambda}_{i j}(t)\right. \\
& \left.-\sum_{\alpha \beta} \Lambda_{i j}^{\alpha \beta}(t)\left(\gamma_{i}^{\alpha \mu}(t) \breve{g}_{i}^{\beta}(t)+\gamma_{i}^{\beta \mu}(t) \breve{g}_{i}^{\alpha}(t)\right)\right) \\
& -\sum_{\beta} a_{j}^{\mu \beta}(t) \check{f}_{1 j}^{\beta}(t)+\sum_{\alpha \beta} Q_{j}^{\alpha \beta}(t)\left(\gamma_{j}^{\alpha \mu}(t) \breve{f}_{j}^{\beta}(t)+\gamma_{j}^{\beta \mu}(t) \breve{f}_{j}^{\alpha}(t)\right) .
\end{aligned}
$$

Dropping Greek indices and using matrix form result in Eq. (21).

\section{A.2. Second Central Moment}

\section{Integrating}

$$
\begin{aligned}
\frac{d \gamma_{j}^{\mu \nu}(t)}{d t}= & \sum_{i} \frac{\pi_{i}(t)}{\pi_{j}(t)} \int x_{j}^{\mu} x_{j}^{\nu}\left(\lambda_{i j}(y, t) f(y \mid i, t)-\bar{\lambda}_{i j}(t) f(y \mid j, t)\right) d y \\
& -\int x_{j}^{\mu} x_{j}^{\nu} \frac{\partial A_{j}^{\alpha}(y, t) f(y \mid j, t)}{\partial y^{\alpha}} d y+\frac{B_{j}^{\alpha \beta}(t)}{2} \int x_{j}^{\mu} x_{j}^{\nu} \frac{\partial^{2} f(y \mid j, t)}{\partial y^{\alpha} \partial y^{\beta}} d y \\
& +\int x_{j}^{\mu} x_{j}^{\nu}\left(\bar{\mu}_{j}(t)-\mu_{j}(y, t)\right) f(y \mid j, t) d y .
\end{aligned}
$$

Calculate integrals

$$
\begin{aligned}
& \int x_{j}^{\mu} x_{j}^{\nu}\left(\lambda_{i j}(y, t) f(y \mid i, t)-\bar{\lambda}_{i j}(t) f(y \mid j, t)\right) d y \\
& =\int x_{j}^{\mu} x_{j}^{\nu}\left(\lambda_{0 i j}(t) f(y \mid i, t)-\bar{\lambda}_{i j}(t) f(y \mid j, t)\right) d y+\sum_{\alpha \beta} \Lambda_{i j}^{\alpha \beta}(t) \\
& \quad \times \int\left(x_{i}^{\mu}+m_{i j}^{\mu}(t)\right)\left(x_{i}^{\nu}+m_{i j}^{\nu}(t)\right)\left(x_{i}^{\alpha}-\check{g}_{i}^{\alpha}(t)\right)\left(x_{i}^{\beta}-\check{g}_{i}^{\beta}(t)\right) f(y \mid i, t) d y \\
& =\lambda_{0 i j}(t)\left(\gamma_{i}^{\mu \nu}(t)+m_{i j}^{\mu}(t) m_{i j}^{\nu}(t)\right)-\bar{\lambda}_{i j}(t) \gamma_{j}^{\mu \nu}(t)+\sum_{\alpha \beta} \Lambda_{i j}^{\alpha \beta}(t) \\
& \quad \times\left(\gamma_{i}^{\mu \nu}(t) \gamma_{i}^{\alpha \beta}(t)+\gamma_{i}^{\mu \alpha}(t) \gamma_{i}^{\beta \nu}(t)+\gamma_{i}^{\mu \beta}(t) \gamma_{i}^{\alpha \nu}(t)+\gamma_{i}^{\mu \nu}(t) \check{g}_{i}^{\alpha}(t) \check{g}_{i}^{\beta}(t)\right. \\
& \quad-\gamma_{i}^{\alpha \mu}(t) m_{i j}^{\nu}(t) \check{g}_{i}^{\beta}(t)-\gamma_{i}^{\alpha \nu}(t) m_{i j}^{\mu}(t) \check{g}_{i}^{\beta}(t)-\gamma_{i}^{\beta \mu}(t) m_{i j}^{\nu}(t) \check{g}_{i}^{\alpha}(t) \\
& \left.\quad-\gamma_{i}^{\beta \nu}(t) m_{i j}^{\mu}(t) \check{g}_{i}^{\alpha}(t)+\gamma_{i}^{\alpha \beta}(t) m_{i j}^{\mu}(t) m_{i j}^{\nu}(t)+m_{i j}^{\mu}(t) m_{i j}^{\nu}(t) \check{g}_{i}^{\alpha}(t) \check{g}_{i}^{\beta}(t)\right) \\
& =\bar{\lambda}_{i j}(t)\left(\gamma_{i}^{\mu \nu}(t)+m_{i j}^{\mu}(t) m_{i j}^{\nu}(t)\right)-\bar{\lambda}_{i j}(t) \gamma_{j}^{\mu \nu}(t)+\sum_{\alpha \beta} \Lambda_{i j}^{\alpha \beta}(t) \\
& \quad \times\left(\gamma_{i}^{\mu \alpha}(t) \gamma_{i}^{\beta \nu}(t)+\gamma_{i}^{\mu \beta}(t) \gamma_{i}^{\alpha \nu}(t)-\gamma_{i}^{\alpha \mu}(t) m_{i j}^{\nu}(t) \check{g}_{i}^{\beta}(t)\right. \\
& \left.\quad-\gamma_{i}^{\alpha \nu}(t) m_{i j}^{\mu}(t) \breve{g}_{i}^{\beta}(t)-\gamma_{i}^{\beta \mu}(t) m_{i j}^{\nu}(t) \check{g}_{i}^{\alpha}(t)-\gamma_{i}^{\beta \nu}(t) m_{i j}^{\mu}(t) \check{g}_{i}^{\alpha}(t)\right),
\end{aligned}
$$




$$
\begin{gathered}
\int x_{j}^{\mu} x_{j}^{\nu} \sum_{\alpha} \frac{\partial\left(A_{j}^{\alpha}(y, t) f(y \mid j, t)\right)}{\partial y^{\alpha}} d y \\
=\int x_{j}^{\mu} x_{j}^{\nu} \sum_{\alpha \beta} \frac{\partial\left(a_{j}^{\alpha \beta}(t)\left(x_{j}^{\beta}-\breve{f}_{1 j}^{\beta}(t)\right) f(y \mid j, t)\right)}{\partial x_{j}^{\alpha}} d x_{j} \\
=\sum_{\beta} \int x_{j}^{\mu} x_{j}^{\nu}\left(\frac{\partial\left(a_{j}^{\mu \beta}(t)\left(x_{j}^{\beta}-\breve{f}_{1 j}^{\beta}(t)\right) f(y \mid j, t)\right)}{\partial x_{j}^{\mu}}\right. \\
\left.+\frac{\partial\left(a_{j}^{\nu \beta}\left(x_{j}^{\beta}-\breve{f}_{1 j}^{\beta}(t)\right) f(y \mid j, t)\right)}{\partial x_{j}^{\nu}}\right) d x_{j} \\
=-\sum_{\beta} \int\left(x_{j}^{\mu} a_{j}^{\nu \beta}+x_{j}^{\nu} a_{j}^{\mu \beta}\right)\left(x_{j}^{\beta}-\breve{f}_{1 j}^{\beta}(t)\right) f(y \mid j, t) d x_{j} \\
=-\sum_{\beta}\left(a_{j}^{\mu \beta}(t) \gamma_{j}^{\nu \beta}(t)+a_{j}^{\nu \beta}(t) \gamma_{j}^{\mu \beta}(t)\right) \\
\int y^{\mu} y^{\nu} \sum_{\alpha \beta} B_{j}^{\alpha \beta}(t) \frac{\partial^{2} f(y \mid j, t)}{\partial y^{\alpha} \partial y^{\beta}} d y=2 B_{j}^{\mu \nu}, \\
\left.=x_{j}^{\mu \beta}(t) \gamma_{j}^{\alpha \nu}(t)+\gamma_{j}^{\mu \nu}(t) \breve{f}_{j}^{\alpha}(t) \breve{f}_{j}^{\beta}(t)\right) \\
=\bar{\mu}_{j}(t) \gamma_{j}^{\mu \nu}(t)+\sum_{\alpha \beta} Q_{j}^{\alpha \beta}(t)\left(\gamma_{j}^{\mu \alpha}(t) \gamma_{j}^{\beta \nu}(t)+\gamma_{j}^{\mu \beta}(t) \gamma_{j}^{\alpha \nu}(t)\right) . \\
+\sum_{\alpha \beta} Q_{j}^{\alpha \beta}(t) \int x_{j}^{\mu} x_{j}^{\nu}\left(x_{j}^{\alpha}-\breve{f}_{j}^{\alpha}(t)\right)\left(x_{j}^{\beta}-\breve{f}_{j}^{\beta}(t)\right) f(y \mid j, t) d x \\
=\mu_{0 j}(t) \gamma_{j}^{\mu \nu}(t)+\sum_{\alpha \beta} Q_{j}^{\alpha \beta}(t)\left(\gamma_{j}^{\mu \nu}(t) \gamma_{j}^{\alpha \beta}(t)+\gamma_{j}^{\mu \alpha}(t) \gamma_{j}^{\beta \nu}(t)\right.
\end{gathered}
$$

Combining all together:

$$
\begin{aligned}
\frac{d \gamma_{j}^{\mu \nu}(t)}{d t} & =\sum_{i} \frac{\pi_{i}(t)}{\pi_{j}(t)}\left(\bar{\lambda}_{i j}(t)\left(\gamma_{i}^{\mu \nu}(t)-\gamma_{j}^{\mu \nu}(t)+m_{i j}^{\mu}(t) m_{i j}^{\nu}(t)\right)\right. \\
& +\sum_{\alpha \beta} \Lambda_{i j}^{\alpha \beta}(t)\left(\gamma_{i}^{\mu \alpha}(t) \gamma_{i}^{\beta \nu}(t)+\gamma_{i}^{\mu \beta}(t) \gamma_{i}^{\alpha \nu}(t)-\gamma_{i}^{\alpha \mu}(t) m_{i j}^{\nu}(t) \breve{g}_{i}^{\beta}(t)\right. \\
& \left.\left.-\gamma_{i}^{\alpha \nu}(t) m_{i j}^{\mu}(t) \breve{g}_{i}^{\beta}(t)-\gamma_{i}^{\beta \mu}(t) m_{i j}^{\nu}(t) \breve{g}_{i}^{\alpha}(t)-\gamma_{i}^{\beta \nu}(t) m_{i j}^{\mu}(t) \breve{g}_{i}^{\alpha}(t)\right)\right)
\end{aligned}
$$




$$
\begin{array}{r}
+\sum_{\beta}\left(a_{j}^{\mu \beta}(t) \gamma_{j}^{\nu \beta}(t)+a_{j}^{\nu \beta}(t) \gamma_{j}^{\mu \beta}(t)\right)+B_{j}^{\mu \nu}(t) \\
-\sum_{\alpha \beta} Q_{j}^{\alpha \beta}(t)\left(\gamma_{j}^{\mu \alpha}(t) \gamma_{j}^{\beta \nu}(t)+\gamma_{j}^{\mu \beta}(t) \gamma_{j}^{\alpha \nu}(t)\right) .
\end{array}
$$

Dropping Greek indices and using matrix form result in Eq. (22).

\section{APPENDIX B}

Using the results of Appendix A, we construct the Gaussian approximation for Eq. (39) for Plan 2. The equation in the brief notation is $\left(j=\theta_{t-}\right)$ :

$$
\begin{aligned}
\frac{\partial \widehat{f}(y, t)}{\partial t}= & -\frac{\partial A_{j}(y, t) \widehat{f}(y, t)}{\partial y}+\frac{B_{j}(t)}{2} \frac{\partial^{2} \widehat{f}(y, t)}{\partial y^{2}} \\
& +\widehat{f}(y, t)\left(\sum_{k \neq j}\left(\widehat{\lambda}_{j k}(t)-\lambda_{j k}(y, t)\right)+\widehat{\mu}_{j}(t)-\mu_{j}(y, t)\right) .
\end{aligned}
$$

Our assumptions in the matrix form are

$$
\begin{gathered}
\mu_{j}(y, t)=\mu_{0 j}(t)+\left(x_{j}-\widehat{f}_{j}(t)\right)^{*} Q_{j}(t)\left(x_{j}-\widehat{f}_{j}(t)\right), \\
\lambda_{j k}(y, t)=\lambda_{0 j k}(t)+\left(x_{j}-\widehat{g}_{j}(t)\right)^{*} \Lambda_{j k}(t)\left(x_{j}-\widehat{g}_{j}(t)\right) .
\end{gathered}
$$

Therefore,

$$
\begin{gathered}
\widehat{\mu}_{j}(t)=\mu_{0 j}(t)+\widehat{f}_{j}^{*}(t) Q_{j}(t) \widehat{f}_{j}(t)+\operatorname{Tr}\left(\gamma_{j}(t) Q_{j}(t)\right), \\
\widehat{\lambda}_{j k}(t)=\mu_{0 j k}(t)+\widehat{g}_{j}^{*}(t) \Lambda_{j k}(t) \widehat{g}_{j}(t)+\operatorname{Tr}\left(\gamma_{j}(t) \Lambda_{j k}(t)\right) .
\end{gathered}
$$

The integral evaluation is similar to that performed in Appendix A. Integrals for calculation of the equation for $\widehat{m}_{j}(t)$ are

$$
\begin{gathered}
\int y \frac{\partial\left(A_{j}(y, t) \widehat{f}_{j}(y, t)\right)}{\partial y} d y=a_{j}(t) \widehat{f}_{1 j}(t), \\
\int y \frac{\partial^{2} \widehat{f}_{j}(y, t)}{\partial y^{2}}=0
\end{gathered}
$$




$$
\begin{gathered}
\int y\left(\widehat{\mu}_{j}(t)-\mu_{j}(y, t)\right) \widehat{f}_{j}(y, t) d y=2 \widehat{\gamma}_{j}(t) Q_{j}(t) \widehat{f}_{j}(t), \\
\int y\left(\widehat{\lambda}_{j k}(t)-\lambda_{j k}(y, t)\right) \widehat{f}_{j}(y, t) d y=2 \widehat{\gamma}_{j}(t) \Lambda_{j k}(t) \widehat{g}_{j}(t) .
\end{gathered}
$$

Integrals for calculation of the equation for $\widehat{\gamma}_{j}$ are

$$
\begin{gathered}
\int x_{j} x_{j}^{*} \frac{\partial A_{j}(y, t) \widehat{f}_{j}(y, t)}{\partial y} d y=-\left(a_{j}(t) \widehat{\gamma}_{j}(t)+\widehat{\gamma}_{j}(t) a_{j}^{*}(t)\right), \\
B_{j}(t) \int x_{j} x_{j}^{*} \frac{\partial^{2} \widehat{f}_{j}(y, t)}{\partial y^{2}} d y=2 B_{j}(t), \\
\int x_{j} x_{j}^{*}\left(\widehat{\mu}_{j}(t)-\mu_{j}(y, t)\right) \widehat{f}_{j}(y, t) d y=-2 \widehat{\gamma}_{j}(t) Q_{j}(t) \widehat{\gamma}_{j}(t), \\
\int x_{j} x_{j}^{*}\left(\widehat{\lambda}_{j k}(t)-\lambda_{j k}(y, t)\right) \widehat{f}_{j}(y, t) d y=-2 \widehat{\gamma}_{j}(t) \Lambda_{j k}(t) \widehat{\gamma}_{j}(t) .
\end{gathered}
$$

Expressions for $\widehat{m}_{j}(t)$ and $\widehat{\gamma}_{j}(t)$ are given in Eq. (44) and (45).

\section{APPENDIX C}

Eqs. (46) and (47) are obtained using Eq. (40) which is rewritten as

$$
\widehat{f}\left(y, \tau_{p}\right)=\widehat{f}\left(y, \tau_{p}-\right) \frac{\lambda_{0 k j}\left(\tau_{p}-\right)+\left(x_{k}-\widehat{g}_{k}\left(\tau_{p}-\right)\right)^{*} \Lambda_{k j}\left(\tau_{p}\right)\left(x_{k}-\widehat{g}_{k}\left(\tau_{p}-\right)\right)}{\hat{\lambda}_{k j}\left(\tau_{p}\right)} .
$$

Integration gives

$$
\begin{aligned}
\widehat{m}_{j}\left(\tau_{p}\right)= & \int\left(x_{k}+\widehat{m}_{k}\left(\tau_{p}-\right)\right) \widehat{f}\left(y, \tau_{p}-\right) \\
& \times \frac{\lambda_{0 k j}\left(\tau_{p}-\right)+\left(x_{k}^{*}-\widehat{g}_{k}{ }^{*}\left(\tau_{p}-\right)\right) \Lambda_{k j}\left(\tau_{p}\right)\left(x_{k}-\widehat{g}_{k}\left(\tau_{p}-\right)\right)}{\widehat{\lambda}_{k j}\left(\tau_{p}-\right)} d y \\
= & \widehat{m}_{k}\left(\tau_{p}-\right)-\frac{2 \widehat{\gamma}_{k}\left(\tau_{p}-\right) \Lambda_{k j}\left(\tau_{p}\right) \widehat{g}_{k}\left(\tau_{p}-\right)}{\widehat{\lambda}_{k j}\left(\tau_{p}-\right)} .
\end{aligned}
$$

Similarly,

$$
\widehat{\gamma}_{j}\left(\tau_{p}\right)=\widehat{\gamma}_{k}\left(\tau_{p}-\right)+\frac{2 \widehat{\gamma}_{k}\left(\tau_{p}-\right) \Lambda_{k j}\left(\tau_{p}\right) \widehat{\gamma}_{k}\left(\tau_{p}-\right)}{\widehat{\lambda}_{k j}\left(\tau_{p}-\right)} .
$$




\section{APPENDIX D}

The likelihood function of Eq. (56) for the case of two discrete states (healthy and unhealthy) can be represented as a product of terms corresponding to age interval between two events: $\widehat{L}_{i}^{G}=p\left(\theta^{i}\left(t_{0}\right)\right) \prod_{j}^{n(i)+m(i)} \widehat{L}_{i j}^{G}$. The functional forms of $\widehat{L}_{i j}^{G}$ depend on the type of events forming the age intervals. These events are associated with: i) measurements of physiological state, ii) changes in health status, and iii) transitions to death or censoring. There are five types of such interval-specific contributions:

1. Measurement $\rightarrow$ Measurement: $\quad \widehat{L}_{i j}^{G}=\hat{\chi}\left(y_{t_{j}}, t_{j-1}, t_{j}-\right) S_{c}\left(t_{j-1}, t_{j}\right)$ for $c=H, D$.

2. Measurement $\rightarrow$ Jumping (HD): $\widehat{L}_{i j}^{G}=\hat{\lambda}_{H D}(\tau) S_{H}\left(t_{j}, \tau\right)$.

3. Measurement $\rightarrow$ Death or censoring: $\widehat{L}_{i j}^{G}=\hat{\mu}_{c}(T)^{\delta_{i}} S_{c}\left(t_{j}, T\right)$ for $c=H, D$.

4. Jumping $\rightarrow$ Measurement: $\widehat{L}_{i j}^{G}=\hat{\chi}\left(y_{t_{j}}, \tau, t_{j}-\right) S_{D}\left(\tau, t_{j}\right)$.

5. Jumping $\rightarrow$ Death/censoring: $\widehat{L}_{i j}^{G}=\hat{\mu}_{D}(T)^{\delta_{i}} S_{D}(\tau, T)$.

Here

$$
\begin{gathered}
S_{H}\left(t_{j-1}, t_{j}\right)=\exp \left(-\int_{t_{j-1}}^{t_{j}}\left(\hat{\lambda}_{H D}(t)+\hat{\mu}_{H}(t)\right) d t\right), \\
S_{D}\left(t_{j-1}, t_{j}\right)=\exp \left(-\int_{t_{j-1}}^{t_{j}} \hat{\mu}_{D}(t) d t\right), \\
S_{D}\left(\tau, t_{j}\right)=\exp \left(-\int_{\tau}^{t_{j}} \hat{\mu}_{D}(t) d t\right), \\
S_{H}\left(t_{j}, \tau\right)=\exp \left(-\int_{t_{j}}^{\tau}\left(\hat{\lambda}_{H D}(t)+\hat{\mu}_{H}(t)\right) d t\right), \\
\hat{\chi}\left(y_{t_{j}}, u, t_{j}-\right)=\left(2 \pi\left|\hat{\gamma}\left(t_{j}-\right)\right|\right)^{-\frac{1}{2}} \exp \left(-\frac{\left(y_{t_{j}}-\hat{m}\left(t_{j}-\right)\right)^{2}}{2 \hat{\gamma}\left(t_{j}-\right)}\right),
\end{gathered}
$$

where $u=t_{j-1}$ for the type 1 and $u=\tau$ for the type 4 . In both cases $\hat{m}(t)$ and $\hat{\gamma}(t)$ are obtained as solutions of Eq. (44) and (45). For the type 1 the initial conditions are $\hat{m}\left(t_{j-1}\right)=y_{t_{j-1}}, \hat{\gamma}\left(t_{j-1}\right)=0$, and for the type 4 they are $\hat{m}(\tau)=\hat{m}(\tau-)-\frac{2 \hat{\gamma}(\tau-) \Lambda_{H D}(g(\tau)-m(\tau-))}{\hat{\lambda}_{H D}(\tau-)}$ and $\hat{\gamma}(\tau)=\hat{\gamma}(\tau-)+\frac{2 \hat{\gamma}^{2}(\tau-) \Lambda_{H D}(\tau-)}{\hat{\lambda}_{H D}(\tau-)}$. 
Copyright of Mathematical Population Studies is the property of Routledge and its content may not be copied or emailed to multiple sites or posted to a listserv without the copyright holder's express written permission.

However, users may print, download, or email articles for individual use. 